\title{
La régulation des canaux d'irrigation : caractérisation et classification
}

\author{
Pierre-Olivier Malaterre \\ Ingénieur-Chercheur à la Division Irrigation du CEMAGREF Montpellier \\ 361, Rue J.-F. Breton, B.P. 5095, 34033 Montpellier Cedex 1 \\ Tél. : 67046356 - Fax : 67635795
}

De nombreuses méthodes de régulation des canaux d'irrigation ont été mises au point de par le monde. Ces méthodes diffèrent d'un pays à un autre, d'une région à une autre. Elles vont des plus sommaires, développées il y a plus de 2000 ans, aux plus sophistiquées mises au point récemment, ou en cours de développement, à partir des techniques modernes de l'automatique. Une littérature importante existe sur le sujet. Cependant, les terminologies employées par les différents auteurs portent encore à confusion et bien que diverses tentatives de classification aient été faites, aucune ne parait vraiment satisfaisante.

Le premier chapitre de cet article s'attache à présenter le contexte général de l'irrigation, et permet de définir ce que l'on entend par « régulation des canaux d'irrigation ". Le second chapitre présente l'esprit des différentes classifications existantes et montre en quoi elles sont insuffisantes. Le troisième chapitre tente de combler cette lacune en définissant une caractérisation des méthodes de régulation basée sur quatre critères essentiels, et en définissant toute classification en référence à ces quatre critères. Le chapitre de conclusion illustre l'utilisation de cette caractérisation, qui peut conduire à diverses classifications en fonction du critère et de la loi de partition choisis. Cette illustration est facilitée par l'utilisation d'un tableau regroupant les caractérisations des diverses méthodes de régulation trouvées dans la littérature.

Les idées maîtresses de cet article sont issues d'une Thèse de Doctorat (Malaterre 1994). L'essentiel de ce document a également été présenté lors d'une réunion d'un groupe de travail de l'American Society of Civil Engineers sur la régulation des canaux d'irrigation (ASCE Task Committee on "classification of and comparison criteria for canal automation algorithms ») tenue le 4 Novembre 1993 à l'US Water Conservation Laboratory (Phoenix, Arizona, USA). Le but de ce groupe de travail, auquel le CEMAGREF participe, est de faire le point sur les différentes méthodes de régulation des canaux existantes dans le monde.

\section{CONTEXTE GÉNÉRAL}

\subsection{L'eau et l'irrigation}

L'eau est une ressource rare. Bien que renouvelable, elle existe en quantités limitées, avec de fortes hétérogénéités dans l'espace et dans le temps. La gestion de cette ressource a depuis toujours été l'objet de l'attention de l'homme. Elle est devenue de plus en plus exigeante et délicate du fait de la compétition croissante existant entre ses différents utilisateurs : agriculture, industrie, besoins domestiques, et environnement (Valiron 1984, Hubert 1984). En France, l'agriculture consomme, à elle seule, plus de $36 \%$ de cette ressource (fig. I), l'industrie $22 \%$ et les usages domestiques $9 \%$ (chiffres 1988, sources Ministère de l'Environnement, cité par Lesaffre et al. 1992). L'irrigation représente plus de $80 \%$ de la consommation en eau dans le monde (chiffres 1975, cité par ASCE 1993 ,

\section{Irrigation canals regulation : characterisation and classification}

This article suggests and defines a characterisation of irrigation canal regulation methods, based on four criteria: considered variables (controlled, measured, and control), logic of control, synthesis method (architecture and technique) and implementation (architecture and device). It is then possible, using this characterisation, to define different classifications, in function of the chosen criterion and partition rule. In conclusion, this article presents a structured characterisation table, containing the main methods of canal regulation found in the literature. 


\begin{tabular}{|c|c|}
\hline \multicolumn{2}{|r|}{ SIGLES ET ABRÉVIATIONS } \\
\hline ASCE & American Society of Civil Engineers \\
\hline $\begin{array}{l}B F \\
B F\end{array}$ & $\begin{array}{l}\text { Boucle Fermée } \\
\text { Boucle Fermée par l'amont }\end{array}$ \\
\hline $\begin{array}{l}B r a m \\
B F\end{array}$ & Boucle Fermée par l'aval \\
\hline$B F_{m i}^{a v}$ & Boucle Fermée mixte \\
\hline $\begin{array}{l}\text { BO } \\
\text { CACG }\end{array}$ & $\begin{array}{l}\text { Boucle Ouverte } \\
\text { Compagnie d'Aménagement des Co- } \\
\text { teaux de Gascogne }\end{array}$ \\
\hline CARA & $\begin{array}{l}\text { Compagnie d'Aménagement Rural } \\
\text { d'Aquitaine }\end{array}$ \\
\hline CARDD & $\begin{array}{l}\text { Canal Automation for Rapid Demand } \\
\text { Deliveries }\end{array}$ \\
\hline CEMAGREF & $\begin{array}{l}\text { Centre National du Machinisme Agri- } \\
\text { cole, du Génie Rural, des Eaux et des } \\
\text { Forêts }\end{array}$ \\
\hline CNABRL & $\begin{array}{l}\text { Compagnie Nationale d'Aménagement } \\
\text { du Bas-Rhône Languedoc }\end{array}$ \\
\hline $\begin{array}{l}\text { ELFLO } \\
\text { GPC }\end{array}$ & $\begin{array}{l}\text { Electronic Filter and Level Offset } \\
\text { Generalized Predictive Control }\end{array}$ \\
\hline LQR & Linear Quadratic Regulator \\
\hline MIMO & $\begin{array}{l}\text { Multiple Inputs, Multiple Outputs (plu- } \\
\text { sieurs entrées, plusieurs sorties) }\end{array}$ \\
\hline $\mathrm{nl} \mathrm{mO}$ & $\begin{array}{l}\text { n Inputs, } m \text { Outputs ( } \mathrm{n} \text { entrées, } \mathrm{m} \text { sor- } \\
\text { ties) }\end{array}$ \\
\hline PID & $\begin{array}{l}\text { Proportionnel, Intégral, Dérivé (Contrô- } \\
\text { leur) }\end{array}$ \\
\hline PIR & $\begin{array}{l}\text { Proportionnel, Intégral, Retard } \\
\text { (Contrôleur) }\end{array}$ \\
\hline$Q$ & Débit dans le canal \\
\hline $\bar{Q}_{a m}$ & $\begin{array}{l}\text { Débit amont dans le canal } \\
\text { Débit aval dans le canal }\end{array}$ \\
\hline$Q_{\text {in }}^{a}$ & $\begin{array}{l}\text { Débit intermédiaire dans le canal } \\
\text { Société du Canal de Provence }\end{array}$ \\
\hline SISO & $\begin{array}{l}\text { Single Input, Single Output (1 entrée, } 1 \\
\text { sortie) }\end{array}$ \\
\hline$U$ & Variable de contrôle \\
\hline $\begin{array}{l}\text { USBR } \\
V\end{array}$ & $\begin{array}{l}\text { United State Bureau of Reclamation } \\
\text { Volume dans un bief du canal }\end{array}$ \\
\hline w & Ouverture de la vanne du régulateur \\
\hline$Y$ & Variable contrôlée \\
\hline$y$ & Cote de l'eau \\
\hline$y_{a m}$ & $\begin{array}{l}\text { Cote de l'eau amont du bief (donc aval } \\
\text { de la vanne) }\end{array}$ \\
\hline$y_{a v}$ & $\begin{array}{l}\text { Cote de l'eau aval du bief (donc amont } \\
\text { de la vanne) }\end{array}$ \\
\hline$y_{\text {in }}$ & $\begin{array}{l}\text { Cote de l'eau intermédiaire dans le bief } \\
\text { Variable mesurée }\end{array}$ \\
\hline
\end{tabular}

confirmés par Plusquellec et al. 1994, p. 2) et plus de $90 \%$ dans l'ouest des USA (chiffres 1984, cité par ASCE 1993).

La surface totale cultivée dans le monde est d'environ 1500 millions d'hectares (Mha), dont 800 en pays en voie de développement (fig. 2) et 700 en pays industrialisés (Schultze 1989). Sur cette surface totale cultivée, environ 280 Mha sont irrigables, soit trois fois plus qu'il y a quarante ans (Lesaffre et al. 1992). Cette irrigation permet d'augmenter les rendements agricoles et de diminuer l'impact des aléas ou déficiences climatiques.

Une part importante (environ 200 Mha, soit $70 \%$ ) de cette surface irrigable est située dans les pays en voie de développement où elle représente $25 \%$ des terres cultivées. Les $30 \%$ restants (environ $80 \mathrm{Mha}$ ) sont situés dans les pays industrialisés où ils représentent $11 \%$ des terres cultivées. Dans les deux cas, les terres irrigables contribuent très fortement à la production agricole.

L'irrigation revêt donc une importance particulièrement grande dans les pays en voie de développement, où les objectifs de production alimentaire sont prioritaires. Dans les pays industrialisés, la recherche de rendements élevés

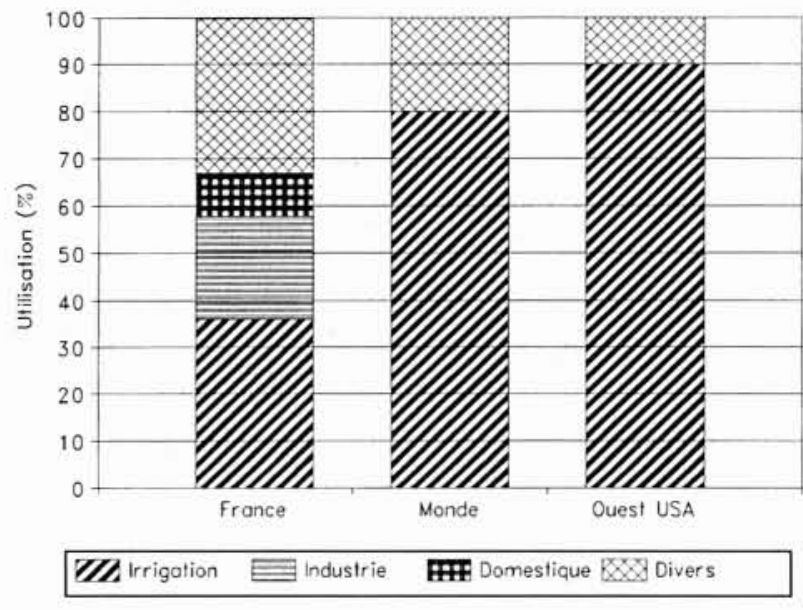

1. Répartition de l'utilisation de l'eau par secteur.

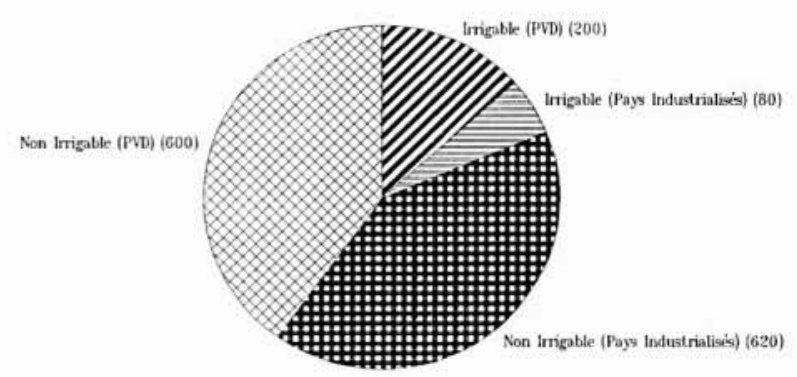

2. Répartition des surfaces cultivées (en millions d'hectares).

n'est plus une priorité. Par contre, l'efficacité de l'utilisation de l'eau doit être améliorée compte tenu de l'accroissement de la compétitivité entre ses différents utilisateurs, et notamment des contraintes grandissantes liées au respect de l'environnement. De plus, la qualité du service aux utilisateurs est appelée à augmenter.

\subsection{Les canaux d'irrigation}

L'acheminement de l'eau d'irrigation, de sa source (un barrage, une rivière) vers ses usagers (parcelles agricoles, stations de pompage), se fait généralement à travers un réseau complexe et étendu de canaux. Le terme «canal d'irrigation » est utilisé par la suite pour décrire un tel système, en incluant les systèmes multi-utilisateurs et les rivières naturelles (les canaux d'irrigation ont souvent des usages multiples, les principaux consommateurs étant les irrigants). Les rivières d'irrigation ont une infrastructure et des contraintes différentes mais des objectifs de fonctionnement communs. Les rivières gérées par la CACG ${ }^{(1)}$ ou la CARA $\left({ }^{2}\right)$ en sont un exemple.

(') Compagnie d'Aménagement des Coteaux de Gascogne.

(2) Compagnie d'Aménagement Rural d'Aquitaine 
La gestion, au sens large, d'un canal d'irrigation comprend des composantes techniques, financières, socio-économiques, et politiques (fig. 3). Sous son aspect technique, la gestion d'un canal d'irrigation est caractérisée par:

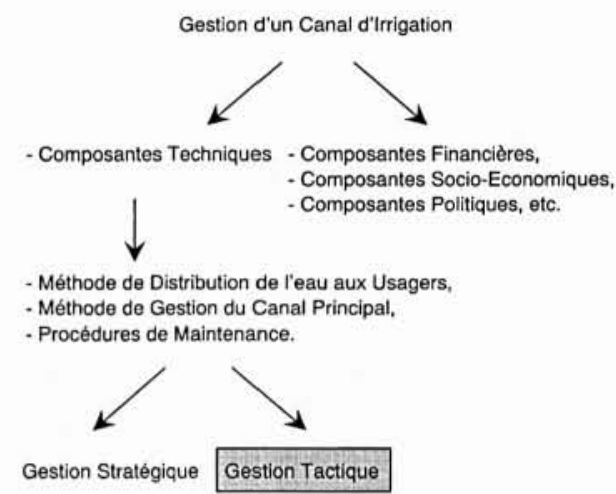

\section{La gestion d'un canal d'irrigation.}

- une méthode de distribution de l'eau aux usagers,

- une méthode de gestion du canal principal,

- des procédures de maintenance.

Cette gestion technique interagit fortement avec les autres composantes de la gestion (financières, socioéconomiques, et politiques). D'autre part, l'infrastructure physique impose des contraintes sur les procédures ou méthodes de gestion envisageables. Dans les chapitres qui suivent, nous présentons ces aspects et illustrons les contraintes correspondantes. Nous détaillons plus particulièrement la «gestion du canal principal », en introduisant les notions de "gestion stratégique " et de "gestion tactique ».

\subsubsection{Méthodes de distribution de l'eau aux usagers}

Différentes méthodes de distribution de l'eau aux usagers sont décrites dans la littérature (Clemmens 1987, Merriam 1992, Goussard 1993, Plusquellec et al. 1994). Ces méthodes se distinguent suivant la liberté laissée aux irrigants dans le choix des caractéristiques débit-durée-fréquence de leurs irrigations.

- La distribution « à la demande " ( limited rate demand schedule») permet aux irrigants de prélever l'eau lorsqu'ils la désirent, sans demande préalable (débit, durée, et fréquence libres). Seule la valeur du débit maximum est fixée, en fonction du matériel utilisé et des infrastructures existantes. Cette méthode est appliquée au "Canal de Provence », France et au «Tranquillity Irrigation District », San Joaquin Valley, Californie, USA. Une telle méthode procure une grande qualité de service, en libérant les irrigants de contraintes horaires. En théorie, une telle souplesse de distribution permet d'optimiser l'efficience de l'utilisation de l'eau à la parcelle, en adaptant au mieux les irrigations aux besoins réels des cultures (Bandaragoda 1992).

- La distribution « au tour d'eau » ("rotation schedule») contraint les irrigants à prélever l'eau selon un calendrier de distribution préétabli (débit, durée, et fréquence fixés).
Cette méthode est très répandue dans le monde, surtout dans les pays en voie de développement. Elle est la plus simple pour le gestionnaire, mais la plus contraignante pour les usagers. Elle est également la plus économique, dans la mesure où elle minimise le débit maximum requis dans le canal principal. En période de pénurie elle permet de limiter et de contrôler les consommations. Sa rigidité conduit cependant très fréquemment à de faibles efficiences à la parcelle, car l'eau n'est pas toujours distribuée au moment optimal.

De nombreuses variantes existent entre ces deux méthodes extrêmes, suivant le degré de liberté accordé aux caractéristiques débit-durée-fréquence de la distribution (ex. : «central scheduling », « arranged schedule», etc., avec des définitions parfois différentes entre auteurs). Plus une méthode de distribution est souple, plus les perturbations créées sur le système par les usagers sont a priori importantes et imprévisibles. Les méthodes de gestion (stratégique et tactique définies ci-dessous) d'un canal sont ainsi directement liées à la méthode de distribution choisie.

\subsubsection{Méthodes de gestion du canal principal}

Dans la suite, nous focalisons l'étude sur les méthodes de gestion du canal principal. Cette gestion comprend deux niveaux de prise de décision : la gestion stratégique et la gestion tactique (Trouvat 1991, Plusquellec et al. 1994, p. 23).

- La gestion stratégique, ou programmation d'allocation de l'eau ( scheduling of water deliveries »), concerne le système global: ressource, canaux, et usagers. Elle consiste à définir une stratégie d'allocation de l'eau en fonction des ressources disponibles, des besoins éventuellement affectés de priorités, des contraintes techniques liées à l'infrastructure existante (Fang et al. 1989, Parent 1991) et des contraintes de programmation liées à la méthode de distribution choisie. Son pas de temps est de l'ordre de la semaine.

- La gestion tactique, ou régulation («water control», "canal control», ou « regulation»), concerne le canal d'irrigation et ses ouvrages. Elle vise à satisfaire les objectifs de distribution définis par la gestion stratégique. Son pas de temps varie de quelques minutes à quelques heures. Par la suite, pour simplifier la terminologie employée, le terme de gestion s'applique à la gestion tactique. Afin d'assurer une gestion correcte (dans l'espace et dans le temps), le gestionnaire a la charge de manœuvrer les ouvrages mobiles construits le long du canal dont il est responsable. Ces ouvrages (vannes, seuils) permettent de modifier des pertes de charge locales, et par conséquent de contrôler indirectement les niveaux, débits ou volumes dans le canal. Les contraintes relatives aux méthodes de régulation sont fréquemment évoquées dans la littérature (Kraatz et al. 1982, Plusquellec 1988, Buyalski et al. 1991, Valera 1991, Goussard 1993, Plusquellec et al. 1994). En particulier, certaines méthodes nécessitent des berges horizontales sur la totalité ou une partie de chaque bief, alors que d'autres sont compatibles avec des berges parallèles au fond du canal. En outre, ces méthodes de régulation ont des comportements différents du fait de l'existence ou non de volumes de stockage intermédiaires.

\subsubsection{Procédures de maintenance}

La maintenance d'un canal est essentielle à la qualité de son fonctionnement et à la pérennité de ses performances 
(Verdier et al. 1992). Le niveau technique requis pour assurer la maintenance des ouvrages de régulation d'un canal dépend des méthodes utilisées et, plus particulièrement, des choix technologiques de mise en œuvre. Ainsi, les méthodes modernes de régulation automatique développées en France et aux Etats-Unis sont encore difficilement applicables aux pays en voie de développement. D'autre part, lorsque l'eau d'irrigation est très chargée en sédiments, certaines méthodes de régulation, facilitant les dépôts solides, sont à proscrire.

\subsection{L'automatisation}

Différentes contraintes techniques (complexité du fonctionnement des ouvrages, interactions, temps de retard, non linéarités) et de fonctionnement (risques de débordement, stabilité des berges, niveaux d'alimentation des prises) rendent la gestion des canaux difficile. De ce fait, la qualité de distribution des canaux traditionnels gérés manuellement est souvent très médiocre, pour des périmètres fonctionnant bien en deçà de leur potentiel :

- les rendements obtenus (pour le riz) sont de l'ordre de 1.5 à 2 tonnes par hectare au lieu des 6 considérés comme réalisables (Plusquellec 1988),

- l'efficience (rapport entre l'eau nécessaire aux cultures et l'eau prélevée dans le barrage) est de l'ordre de $30 \%$ (ou inférieure) alors que $60 \%$ est considéré comme réalisable (Plusquellec et al. 1994, p. 55). Une étude du Ministère de l'Intérieur Américain, réalisée sur plus de 60 périmètres irrigués situés aux USA, estime que l'efficience moyenne est de $44 \%$, et qu'une meilleure gestion du système de transport et de distribution principal permettrait d'atteindre une valeur de $61 \%$ (Rapport 1978, cité par ASCE 1993).

En outre, les conditions de fonctionnement sont parfois sources de conflits :

- la distribution au tour d'eau est contraignante pour les usagers,

- il existe des iniquités de distribution entre les usagers situés à l'amont et ceux situés à l'aval.

Des recherches sont menées actuellement pour améliorer la qualité de la gestion de ces canaux, en conservant les structures existantes et la gestion manuelle (IIMI 1989, Malaterre 1989, Bhutta et al. 1992, Kosuth et al. 1992, Baume et al. 1992b et 1993, Rey et al. 1993).

Lorsque les conditions socio-économiques le permettent (disponibilité d'une main d'œuvre qualifiée, réseaux électrique et téléphonique fiables, pièces de rechange disponibles, non dégradation des installations par les usagers, etc.), l'automatisation des ouvrages est une voie possible d'amélioration de la régulation d'un canal d'irrigation. Plusieurs niveaux d'automatisation existent (Goussard 1993) :

- surveillance-acquisition automatique,

- télécommande automatique,

- élaboration automatique des consignes et/ou des ordres,

- automatisme de manœuvre,

- automatisme de réglage.
Les avantages de l'automatisation des systèmes d'irrigation sont nombreux (Baudequin et al. dans Framji et al. 1978) :

- avantages financiers,

- économie d'eau,

— diminution de la main d'œuvre,

- économie sur les coûts d'investissement (réservoirs tampons),

- économie sur les coûts de fonctionnement (personnel), - utilisation optimale de l'eau disponible, dans un contexte de compétitivité de plus en plus forte entre usagers,

- qualité de service, vers une tendance de distribution à la demande,

- facilité de gestion et d'acquisition d'information.

Remarque : le coût additionnel lié à l'automatisation d'un canal d'irrigation à été évalué à environ $3 \%$ du coût total d'une conception traditionnelle (Plusquellec et al. 1994, p. 67).

Ces avantages expliquent les efforts consacrés en recherche et développement, depuis les années 1960-1970, pour le contrôle automatique des canaux d'irrigation. Les méthodes développées sont basées sur des actionneurs mécaniques, hydropneumatiques, ou électromécaniques. Une évolution semblable a lieu dans d'autres domaines de la gestion de l'eau, tels que l'assainissement, l'adduction d'eau potable ou la protection contre les crues (Frerot 1987, Gilbert 1990, Valiron 1988). Cependant, ces applications sont encore limitées au regard des applications de l'automatique au domaine industriel (chimie, génie des procédés, électricité, spatial, etc.).

\section{II $\square$ CRITIQUE BIBLIOGRAPHIQUE}

De nombreux auteurs ont tenté de classifier les différentes méthodes de régulation des canaux mises au point de par le monde (Hussou et al. dans Framji et al. 1978, De Leon 1986, Zimbelman 1987, Buyalski et al. 1991, Chevereau 1991, Schuurmans 1991, Malaterre 1991, Deltour 1988 et 1992, Baume et al. 1990 et 1992a, Khaladi 1992, Ankum 1992, 1993a et 1993b, Goussard 1993, Plusquellec et al. 1988 et 1994). Les termes utilisés par ces auteurs, souvent hydrauliciens de formation, se réfèrent à des concepts parfois mal définis et différents de nature (commande proportionnelle, commande par l'amont ou par l'aval, commande à niveau aval, à niveau amont, à débit ou à volume constant, commande répartie ou centralisée, commande manuelle, automatique mécanique ou automatique électronique, etc.). Les définitions utilisées sont différentes voire contradictoires entre auteurs (Ankum 1992 et 1993, Goussard 1993, Chevereau 1991).

A titre d'exemple Ankum (1992 et 1993) propose la classification suivante :

- commande proportionnelle,

- commande par l'amont,

- commande par l'aval,

- commande à volume constant ou BIVAL,

- commande ELFLO,

- commande CARDD.

Plusquellec et al. (1994) proposent, quant à eux :

- commande proportionnelle,

- commande modulable en débit,

- commande par l'amont, 
- commande par l'aval,

- télésurveillance,

- télécontrôle.

Ces deux «classifications », très représentatives de ce que l'on trouve habituellement dans la littérature, sont plus des listes de méthodes ou de réalisations rencontrées sur le terrain, que de réelles classifications définies et structurées. En effet, les classes définies font référence à plusieurs critères différents, et sont de ce fait non disjointes. Par exemple, les méthodes ELFLO et CARDD sont également des méthodes de commande par l'aval, et des méthodes télécontrôlées peuvent être également par l'amont ou par l'aval (ex.: Régulation Dynamique de la Société du Canal de Provence).

Goussard (1993) propose une classification plus satisfaisante des méthodes de régulation, basée sur leur architecture de mise en cuvre :

- la régulation répartie :

- régulation par l'amont,

- régulation par l'aval,

- régulation à niveaux associés,

- régulation mixte,

- la régulation centralisée :

- programmée,

- temps réel,

- la régulation répartie sous contrôle centralisé.

Cependant, cette classification présente le défaut de définir les régulations par l'amont et par l'aval comme des sous-classes de la régulation répartie. Or, comme le souligne d'ailleurs l'auteur, les concepts de régulation par l'amont ou par l'aval peuvent également être appliqués aux régulations centralisées. Ceci provient du fait que la classification est basée sur deux critères différents, qu'il conviendrait de dissocier : l'architecture de mise en œuvre et la logique de contrôle.

Chevereau (1991) souligne l'ambiguïté des classifications existantes, et en présente une, plus satisfaisante dans le cas particulier d'une commande en débit sur un bief unique équipé d'une vanne en tête. Il introduit en particulier la notion essentielle, pour les automaticiens, de «boucle ouverte» et de «boucle fermée ». Cependant, il associe la notion de commande par l'amont avec la notion de commande en boucle ouverte (p. 6, respectivement de commande par l'aval et de commande en boucle fermée, p. 7), ce qui n'est pas exact, dans le cadre plus général d'une régulation quelconque (Cf. les vannes AMIL d'Alsthom Fluide, à niveau amont constant, qui sont des vannes fonctionnant en boucle fermée, et qui sont pourtant caractéristiques d'une commande par l'amont).

Remarque 1 : la littérature fait souvent référence aux régulations "par l'amont» ou " par l'aval». Cette terminologie est source fréquente de confusion. En effet, même si les régulations "par l'aval» (respectivement "par l'amont ») visent traditionnellement (pour des raisons de simplicité technologique) à maintenir une cote constante à l'aval (respectivement à l'amont) immédiat des régulateurs, cela n'est pas forcément le cas. On parle, par exemple, de régulation par l'aval distant lorsque la cote contrôlée est la cote amont du régulateur aval suivant! Nous préférons, dans la classification présentée au chapitre suivant, définir les cotes contrôlées par rapport au bief $\left({ }^{3}\right)$ (amont, milieu ou aval d'un bief) et non pas par rapport au régulateur (amont ou aval d'un régulateur). Nous définirons au chapitre 3.3.2.2. la régulation "par l'amont " et " par l'aval " en tant que logique de contrôle et non pas en référence à une variable contrôlée particulière.

Remarque 2: le terme de "self-management "est utilisé par Ankum (1992 et 1993) pour désigner les systèmes dont la commande en débit est réalisée en boucle fermée. Cette terminologie ambiguë risque d'être confondue avec la notion de « self-regulation » définie par les automaticiens (Shinskey 1988), et n'apporte rien par rapport à la notion bien connue de boucle fermée. Suivant la définition de Shinskey, un système se comporte en auto-régulation (selfregulation), lorsqu'il évolue spontanément vers un état d'équilibre. Cet état d'équilibre peut évidemment être différent suivant les conditions de fonctionnement. Par opposition, un système se comporte en non-auto-régulation (non-self-regulation), lorsqu'il butte sur ses conditions extrêmes dès que la commande n'est pas parfaitement ajustée aux objectifs de sortie. C'est le cas pour un canal fermé à l'aval et alimentant des stations de pompage. En effet, si le débit entrant n'est pas exactement le même que le débit pompé, il y a, à terme, soit débordement soit assèchement total. L'immense majorité des canaux d'irrigation fonctionne en auto-régulation, car les excès d'eau sont évacués vers l'aval et les assèchements sont rares puisque les prélèvements gravitaires deviennent impossibles dès que le niveau d'eau dans le canal principal devient trop bas.

Mises à part ces remarques, essentielles à notre sens, sur les terminologies utilisées et sur les classifications choisies, les ouvrages cités ci-dessus contiennent des informations intéressantes et détaillées sur les différentes méthodes de régulation considérées.

\section{III — CLASSIFICATION}

Nous présentons, dans ce chapitre, une classification des différentes méthodes de régulation d'un système canal ou rivière quelconque, et replaçons les termes utilisés par les hydrauliciens dans le langage plus rigoureux des automaticiens. Cette classification s'applique aussi bien aux méthodes traditionnelles que modernes. Des exemples classiques de méthodes sont donnés au cours de la classification. Les méthodes sont repérées par leur nom lorsqu'elles en ont un, ou par le nom de leur(s) auteur(s), ou par la théorie automatique utilisée. Nous ne les présentons pas en détail. Elles sont abondamment décrites et étudiées par leurs auteurs ou dans des travaux de recherche ou de synthèse (Zimbelman 1987, Buyalski 1991, Chevereau 1991, Goussard 1993). Des références bibliographiques détaillées sont données lors de la première apparition de chaque exemple. Elles ne sont pas reprises par la suite, ou réduites, afin de simplifier la lecture du texte.

\subsection{Définition d'une classification}

Afin de classer les éléments d'un ensemble $E$ il faut :

- définir un critère de caractérisation,

$\left({ }^{3}\right)$ Nous appelons "bief " une portion d'un canal, située entre deux ouvrages de régulation («pool » en anglais). 
- définir une partition de l'ensemble $E$ basée sur ce critère.

Remarque : au sens mathématique du terme (Ramis et al. 1979), on appelle "partition" d'un ensemble E, toute famille de parties de E, non vides, disjointes deux à deux, et dont la réunion redonne l'ensemble E. Chaque élément de E est alors associé à une partie unique de la partition.

Dans notre cas, l'ensemble $E$ considéré est l'ensemble des méthodes de gestion (ou régulation) des canaux, développées de par le monde, qu'elles soient appliquées concrètement ou uniquement à l'état de développement théorique.

La difficulté que présente la classification de cet ensemble provient du fait qu'il existe différentes manières de caractériser ses éléments (ex.: les variables contrôlées, l'architecture de mise en œuvre, la gestion des communications, la méthode de synthèse, la gestion des alarmes, la longueur des biefs régulés, etc.). Parmi tous les critères possibles, on veut retenir ceux, en nombre minimum, permettant de rendre compte du comportement hydraulique, des performances et des contraintes des diverses méthodes de régulation des canaux. Nous choisissons de retenir les quatre critères essentiels suivants :

- les variables considérées (contrôlées, mesurées, et de contrôle),

- la logique de contrôle,

- la méthode de synthèse,

- la mise en cuvre.

Ces différents termes sont définis, commentés et illustrés dans les chapitres qui suivent.

Cet ordre n'est pas un ordre de priorité d'intérêt. Selon son domaine de compétence, chaque personne sera plus ou moins sensible à un ou plusieurs des quatre critères évoqués. Ainsi, le gestionnaire et l'ingénieur hydraulicien seront plus sensibles aux variables contrôlées et à la logique de contrôle. L'automaticien sera sensible à la logique de contrôle et à la méthode de synthèse. Le gestionnaire et l'ingénieur chargé de la réalisation des travaux, s'intéresseront aux variables mesurées et à la mise en œuvre.

\subsection{Les variables considérées}

En automatique, un système est usuellement représenté sous la forme figure 4:

où :

- $U$ sont les variables de contrôle du système, sur lesquelles on peut agir, afin de modifier l'état du système,

- $Y$ sont les variables contrôlées du système, auxquelles on peut assigner des consignes (constantes ou variables).

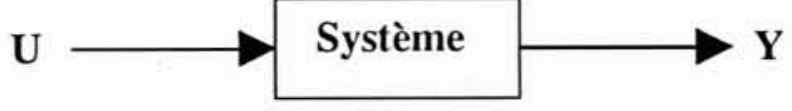

4. Représentation usuelle d'un système.

Remarque: nous définirons au chapitre 3.4 .1 les notions plus générales d'entrée et de sortie d'un système ou d'un contrôleur.

Dans la réalité, les variables contrôlées $Y$ (cotes, débits, volumes, combinaisons linéaires de cotes, etc.) ne sont pas toujours obtenues directement à partir du système, mais sont parfois le fruit de calculs effectués (à travers une fonction $g$ ) à partir de variables physiques $Z$ mesurées sur le système (fig. 5).

\section{Exemple}

La méthode BIVAL a pour variable contrôlée la variable $Y=\alpha y_{a m}+(1-\alpha) y_{a v}$, où $\left.\alpha \in\right] 0,1[$. Les variables mesurées $Z$ sont donc les cotes à l'amont $\left(y_{a m}\right)$ et à l'aval $\left(y_{a v}\right)$ du bief considéré.

Nous n'entrerons pas dans le détail du processus complexe de mesure de ces variables $Z$ (une cote peut être obtenue par la mesure du déplacement d'un flotteur via un potentiomètre, par la mesure d'une pression via une cellule piézo-résistive, par la mesure du temps d'aller-retour d'une onde via un capteur ultrason, etc.). Il est cependant important de connaître ces points de mesure $Z$ (leur nature, leur nombre, et leur emplacement).

De même, les commandes $U$ (ouvertures de vanne, variations d'ouverture de vanne, débits, etc.) ne peuvent pas toujours être appliquées directement au système. Ces commandes $U$ doivent alors être traduites (à travers une fonction $f$ ) en commandes élémentaires $V$ (Figure 5) applicables aux organes de commande du système (impulsions électriques de commande d'un moteur, pression d'alimentation d'un vérin hydraulique, etc.).

Remarque: pour simplifier, on ne s'intéresse pas à la relation qui existe entre une ouverture de vanne et le moyen mécanique, pneumatique, électrique, ou autre de l'obtenir. En général des contrôleurs PID performants et à dynamique rapide contrôlent ces asservissements. On néglige donc la dynamique supplémentaire correspondante. De ce fait, les commandes élémentaires $V$ considérées sont uniquement des ouvertures de vannes. On considère ainsi que l'on est capable de positionner une vanne dans une position donnée de manière infiniment rapide et précise (ou avec des biais négligeables devant les autres dynamiques

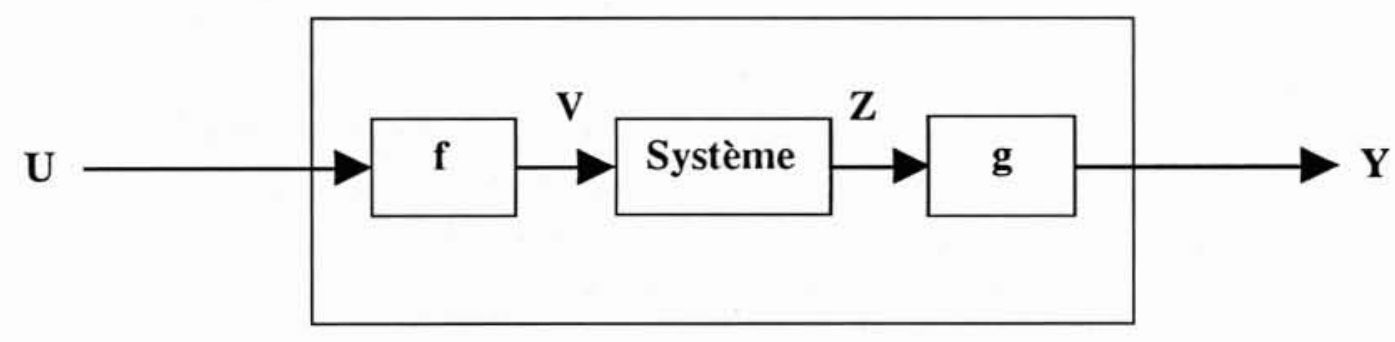

5. Représentation détaillée d'un système. 


\section{RÉGULATION DES CANAUX D'IRRIGATION}

du système). On ne détaillera donc pas, dans les chapitres qui suivent, ces commandes élémentaires V.

Les différentes variables contrôlées $Y$, mesurées $Z$ et de contrôle $U$ sont détaillées ci-dessous.

\subsubsection{Variables contrôlées}

Les variables contrôlées sont les variables du système auxquelles le gestionnaire assigne des consignes. En automatique on parle de « poursuite» (« tracking ») lorsque ces consignes sont variables au cours du temps. Suivant les auteurs, le terme « régulation " est soit général à tout type de consigne (constante ou variable), soit particulier au cas d'une consigne constante nulle. Nous utiliserons le terme « régulation" dans son sens général et le terme «poursuite " dans le cas où l'on veut insister sur la nature variable des consignes.

Les variables hydrauliques contrôlées sur un canal d'irrigation peuvent être de deux natures : des flux (débits) ou des efforts (cotes), selon la terminologie utilisée en modélisation des systèmes physiques par bond-graphs (Borne et al. 1992). Elles peuvent également être des volumes, c'est-à-dire des intégrales de flux (débits) dans le temps, ou d'efforts (cotes) dans l'espace.

\subsubsection{Débits}

Les besoins des différents usagers d'un canal d'irrigation se définissent principalement en terme de débit:

- Besoins agricoles :

— débit délivré à une parcelle,

- débit alimentant un canal secondaire,

- débit prélevé par une station de pompage,

- Besoins pour l'environnement :

- débit restitué en fin de système, ou débit réservé.

Eventuellement, pour des systèmes multi-usages :

- Besoins domestiques :

- débit alimentant une habitation,

- débit prélevé par une station de traitement d'une agglomération,

- Besoins industriels :

- débit prélevé par une usine.

Lorsque des réservoirs de stockage, naturels ou non, sont disponibles (réserve utile du sol, réservoir d'eau, bassin d'une station de traitement, bassin de stockage, volume stocké dans les biefs), les besoins peuvent être définis de manière plus souple, en terme de volume distribué sur une période de temps. Dans ce cas, la variable contrôlée n'est plus une valeur donnée de débit, mais un volume, soit l'intégrale d'un débit sur une période donné. Des fluctuations de débit sont alors autorisées, et amorties par la capacité de stockage des réservoirs. Cependant, ces réservoirs sont coûteux, de tailles limitées et des contraintes de distribution ne suppriment jamais les besoins exprimés en terme de débit.

Par conséquent, tout système hydraulique à surface libre doit être géré, directement ou indirectement, de manière à satisfaire les demandes en débit de ses différents usagers. Compte tenu de la nature du phénomène physique en jeu (écoulement gravitaire de l'amont vers l'aval), ces demandes en débit ne peuvent être satisfaites qu'à partir de la source située à l'amont du système, par vidange des réservoirs amont.

\section{Exemples de régulation}

- GPC $\left(^{4}\right)$ (Sawadogo et al. 1991a, 1991b, 1992a, 1992b

et 1992c, Rodellar et al. 1993),

- CACG (Piquereau et al. 1982 et 1984, Grosclaude et al. 1985, Verdier 1986, Tardieu 1988, Barbet 1990, Rey 1990, Hurand et al. 1993),

- SIMBAK (Chevereau 1991).

\subsubsection{Cotes}

Les débits sont difficilement mesurables en rivières ou canaux à surface libre. Par contre, les cotes peuvent l'être facilement. D'autre part, les contraintes d'alimentation des prises gravitaires, la stabilité des berges des canaux, la lutte contre leur enherbement, la constitution de stocks d'eau intermédiaires, les risques de débordement, etc., créent des contraintes supplémentaires en terme de cote.

Les cotes contrôlées « $y$ » peuvent être situées en amont de bief $\left(y_{a m}, f i g .6\right)$, en aval de bief $\left(y_{a v}, f i g .7\right)$, ou en un point intermédiaire $\left(y_{i n}, f i g .8\right)$. Les caractéristiques de fonctionnement sont très différentes suivant l'option choisie.

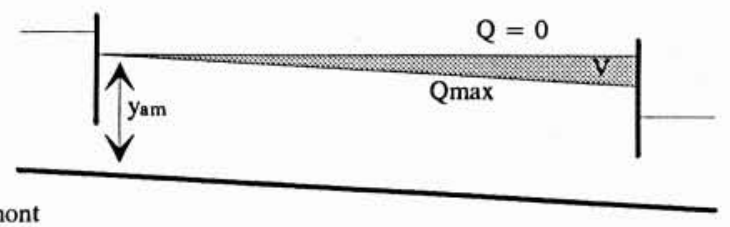

amont

\section{Contrôle d'une cote amont de bief.}

\subsection{Amont de bief}

Avantage : un volume de stockage $V$ est disponible entre le volume à débit nul et le volume à débit maximal. Il permet de répondre rapidement aux demandes imprévues du bief ou de l'aval et de stocker de l'eau en cas de diminution de la consommation.

Inconvénient : les berges du canal doivent être horizontales, ce qui est coûteux en génie civil.

\section{Exemples de régulation}

- Vannes AVIS et AVIO (Mises au point par Alsthom Fluide, Alsthom Fluide 1975-79 et 1981, Goussard 1993), - LittleMan par l'aval (Mis au point par l'USBR (5), existe en version par l'amont et par l'aval, Zimbelman 1987).

\subsection{Aval de bief}

Avantage: les berges du canal peuvent suivre la pente naturelle du terrain, ce qui limite les coûts en génie civil.

Inconvénient : aucun volume de stockage n'est disponible entre le volume à débit nul et le volume à débit maximal. Le système ne peut donc pas répondre rapidement à une demande imprévue. L'eau non consommée ne peut pas être stockée localement et est «perdue » dans les biefs aval.

$\left({ }^{4}\right)$ Generalized Predictive Control.

$\left({ }^{5}\right)$ United State Bureau of Reclamation. 


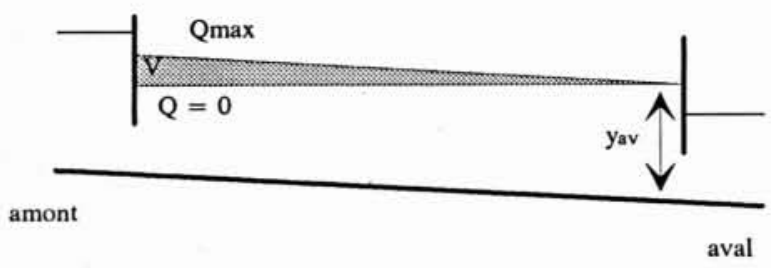

7. Contrôle d'une cote aval de bief.

Remarque : sur les Figures 6 et 7, les cotes contrôlées sont les mêmes à débit nul et à débit maximum. Cela n'est pas toujours le cas (Cf. Vannes Alsthom Fluide). L'écart de cote correspondant est alors appelé "décrément». Un moyen mnémotechnique pour retenir dans quel sens ce décrément est introduit est de noter que les deux courbes (celle pour $Q$ nul et celle pour $Q$ maximum) ne se coupent pas. Le volume de l'onglet mis en jeu est donc augmenté dans les deux cas.

Exemples de régulation

- Vannes AMIL (Mises au point par Alsthom Fluide, Alsthom Fluide 1975-79 et 1981, Goussard 1993),

- ELFLO (6) (Shand 1971, Buyalski et al. 1979),

- CARDD (7) (Burt 1983),

- LittleMan par l'amont (USBR),

- PIR $\left(^{8}\right)$ (Deltour 1992),

- PID ( $\left.{ }^{\circ}\right)$ (UMA Engineering - Imperial Valley, USA),

- PI (SOGREAH - Kirkuk, Irak),

- Zimbelman (Mis au point par Zimbelman en 1981 , Zimbelman 1987).

\subsection{Cote intermédiaire}

Le contrôle d'une cote intermédiaire particulière (voisine du milieu du bief), est équivalent à un contrôle du volume stocké dans le bief. Cette cote peut, soit être mesurée directement (aucun exemple à notre connaissance mais envisageable a priori), soit être obtenue comme combinaison linéaire d'une cote amont et d'une cote aval (ex. : BIVAL).

Avantage : cette méthode présente un compromis entre les deux options précédentes, tant au niveau du coût en génie civil que du volume de stockage disponible V. En effet, les berges ne doivent être horizontales qu'à l'aval de la cote contrôlée.

Inconvénient : une ou plusieurs cotes distantes doivent être mesurées, ce qui implique l'installation de lignes de transmission ou d'un réseau de mesure.

Exemple de régulation

- BIVAL (Mis au point par la SOGREAH, Zimbelman 1987, Chevereau 1991).

\subsubsection{Volumes}

Dans un tel cas, le contrôleur est moins sensible aux parasites, mais les temps de réponse sont augmentés (Hussou et al. dans Framji et al. 1978). Ces méthodes sont

(6) Electronic Filter Level Offset.

( 7 ) Canal Automation for Rapid Demand Deliveries.

( $\left.{ }^{8}\right)$ Proportionnel, Intégral, Retard.

(9) Proportionnel, Intégral, Dérivé.

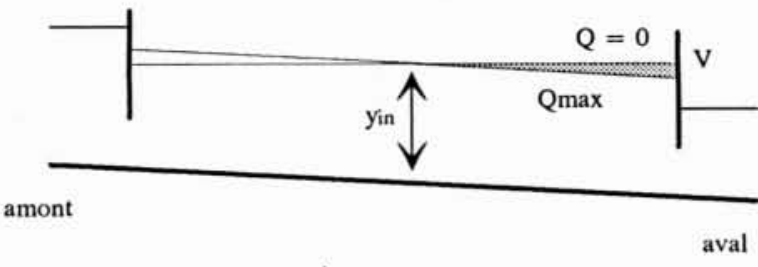

8. Contrôle d'une cote intermédiaire

applicables aux canaux d'irrigation à forts volumes de stockage, et équipés de prises dont l'alimentation n'est pas tributaire des cotes de l'eau dans le canal (ex. : pompages).

Exemples de régulation

- Régulation Dynamique (Mis au point par la SCP $\left({ }^{10}\right)$, Cœuret 1977, Lefebvre 1977, Deltour 1988),

- Controlled Volumes (Mis au point par l'USBR, Buyalski 1991).

\subsubsection{Conclusion}

Les besoins des usagers étant exprimés en terme de débit, les variables hydrauliques contrôlées doivent logiquement être des débits. Cependant, ces derniers sont difficilement mesurables dans les canaux d'irrigation, au contraire des cotes et, dans une moindre mesure, des volumes. De plus, des contraintes de fonctionnement conduisent souvent à considérer des consignes de cotes minimales et maximales, indépendamment des objectifs de satisfaction des besoins des usagers. Des cotes, ou parfois des volumes, sont donc adoptées comme variables contrôlées supplémentaires. L'emplacement des cotes contrôlées est un point important à considérer compte tenu de ses relations avec les coûts en génie civil et les possibilités de stockage d'eau dans les biefs.

Nous verrons, au chapitre 3.3.2, que certaines logiques de contrôle de cote ou de volume conduisent également, de manière indirecte, à un contrôle de débit. Par contre, dans le cas général, le ou les contrôleurs doivent considérer explicitement les deux types de variables:

- les débits, pour satisfaire les besoins des usagers,

- les cotes (ou parfois les volumes), pour satisfaire les contraintes de fonctionnement.

Les variables contrôlées pouvant être de natures différentes, il faut préciser, lorsque l'on parle de commande, s'il s'agit d'une commande en débit, en cote ou en volume.

\subsubsection{Variables mesurées}

Les variables mesurées sur un canal d'irrigation (ou sur une rivière) sont généralement des cotes (ex. : ELFLO, PIR). Dans certains cas, les variables mesurées peuvent également être des débits (ex. : CACG), soit grâce à un équipement spécifique (basé en général sur la mesure d'une ou plusieurs vitesses, ex. : moulinet, débitmètre à ultrason), soit par l'utilisation d'une loi d'ouvrage $Q=f\left(z_{1}, z_{2}, w\right)$, ou d'une courbe de tarage $Q=f(z)$ en une section de contrôle.

(10) Société du Canal de Provence. 
Remarque : à partir du moment où une telle loi $f$ existe, on considère que c'est bien un débit $Q$ qui est mesuré, nonobstant la manière dont ce débit est obtenu, même s'il est calculé à partir d'une ou plusieurs mesures de cote. Ceci est cohérent avec la remarque faite au chapitre 3.2, où nous indiquions ne pas nous intéresser à la manière dont une cote est mesurée, dès que nous estimons qu'une telle mesure est possible, avec une précision considérée comme suffisante.

Enfin, les variables mesurées peuvent être des volumes, évalués grâce à la mesure de plusieurs cotes le long du canal, ou par un bilan de débit entrée-sortie (ex. : Régulation Dynamique).

\subsubsection{Variables de contrôle}

Les variables de contrôle $U$ peuvent être des ouvertures de vanne, des incréments d'ouverture de vanne, des débits, des incréments de débit, etc.

Considérer l'ouverture de vanne comme variable de contrôle présente l'avantage de pouvoir tenir compte, dans le régulateur, de la dynamique complexe liant cette ouverture avec le débit local et les cotes amont et aval. Cette dynamique est importante et il est dommage de ne pas en tenir compte (ex. : LQR CEMAGREF, ELFLO).

Considérer le débit comme variable de contrôle permet de découpler les différents sous-systèmes, ce qui est intéressant lorsque l'on utilise des contrôleurs locaux monovariables (ex. : Régulation Dynamique, PIR). Par contre, on ne tient alors pas compte, dans le contrôleur global, de la dynamique du contrôleur local reliant le débit (commande $U$ ) et l'ouverture de vanne (commande élémentaire $V$ ). On n'est donc pas sûr de la qualité du comportement du contrôleur global puisque certaines dynamiques importantes ont été négligées lors de la synthèse du contrôleur.

Si la variable de contrôle $U$ est un débit, il faut ensuite convertir ce débit en ouverture de vanne $V$, applicable au système. Ceci peut être fait de différentes manières :

- soit par inversion de l'équation statique d'ouvrage $Q=$ $f\left(z_{1}, z_{2}, w\right)$,

- soit par un contrôleur local dynamique, de type PID ou autre.

\subsection{Logiques de contrôle}

\subsubsection{Définitions}

L'automaticien définit clairement la notion de «Boucle Fermée " (BF) ou «Compensation " («Closed Loop » ou «Feedback») et de «Boucle Ouverte" (BO) ou «Anticipation » («Open Loop» ou « Feedforward») suivant la nature des informations utilisées pour calculer les commandes du système.

En boucle fermée, la commande $U$ est calculée à partir de l'erreur mesurée entre la sortie réelle $Y$ (variable contrôlée) et la sortie de consigne $Y_{c}$ (fig. 9). Les perturbations $\mathrm{P}$, même inconnues, sont prises en compte indirectement, à travers leurs effets sur la sortie $\mathrm{Y}$ du système.

En automatique, la notion de boucle fermée est essentielle puisque c'est elle qui permet d'asservir une commande à une consigne. Cependant, pour des procédés complexes (processus à temps mort par exemple, c'est à dire avec des temps de retard, Kuanyi 1989), cette boucle

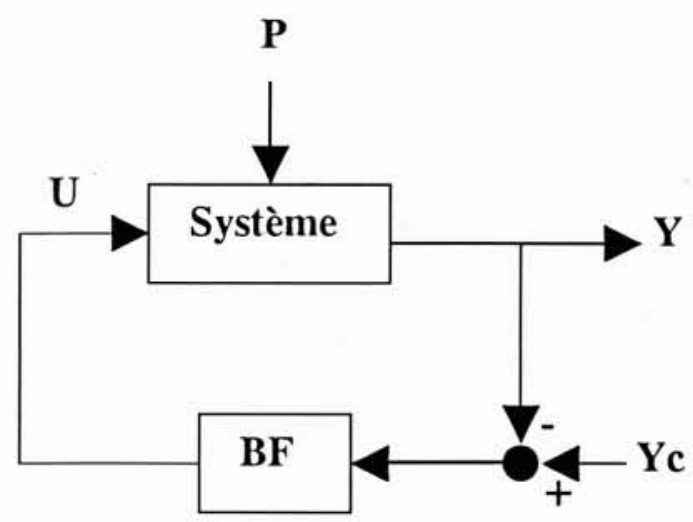

9. Commande en boucle fermée.

fermée ne donne pas entière satisfaction. La qualité du contrôle peut être alors considérablement améliorée par l'adjonction d'une boucle ouverte (Shinskey 1988). Dans le cas des canaux d'irrigation, les temps de retard entre les organes de réglage amont et les débits contrôlés aval sont importants (de quelques minutes à plusieurs heures). Une boucle fermée seule ne peut alors fonctionner correctement que si des volumes de stockage importants sont disponibles. En effet, dans le meilleur des cas la commande aura un retard au moins égal au retard du système. Or, des volumes de stockage impliquent des coûts élevés en génie civil.

En boucle ouverte, la commande $U$ est calculée à partir de la connaissance de la dynamique du système (modèle), des consignes $Y_{c}$ et éventuellement d'une estimation des perturbations $\hat{P}$ (fig. 10). La boucle ouverte peut compenser les temps de retard inhérents au système en anticipant sur les besoins des usagers. Ces besoins doivent être estimés de manière aussi précise que possible, en fonction de données climatiques, agronomiques, sociologiques, et d'enregistrements des consommations des semaines ou saisons passées (Perrin 1989).

Cependant, la boucle ouverte n'est généralement pas suffisante, du fait des erreurs de modèle, des erreurs d'estimation des perturbations, et de l'existence de perturbations inconnues. C'est pour ces raisons que l'association boucle ouverte + boucle fermée est souvent utilisée (fig. II), la boucle fermée permettant de compenser les erreurs de la boucle ouverte.

Dans le cas d'un système multivariable (avec plusieurs variables de commande et plusieurs variables contrôlées), plusieurs boucles de régulation et plusieurs contrôleurs de logiques différentes peuvent être combinés. Par exemple, dans le cas des canaux d'irrigation, les débits peuvent être contrôlés en boucle ouverte et les cotes en boucle fermée.

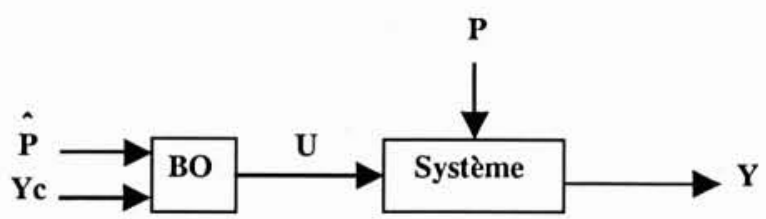

10. Commande en boucle ouverte. 


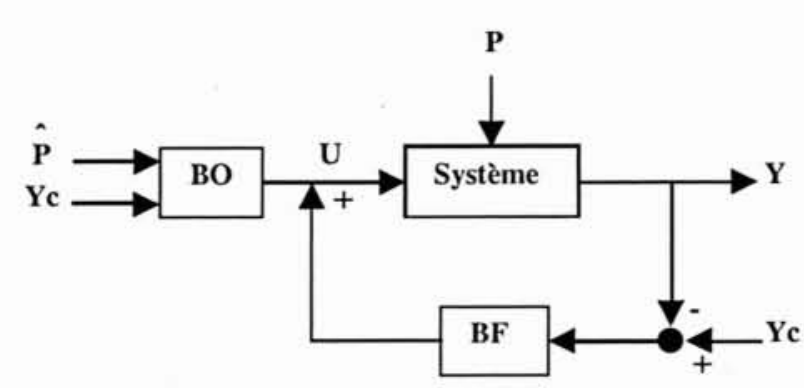

11. Commande en boucle ouverte + boucle fermée.

Certaines méthodes de régulation, citées par la suite, peuvent donc apparaître dans plusieurs catégories.

Nous illustrons, dans les chapitres qui suivent, ces notions de boucle fermée et de boucle ouverte suivant que la variable contrôlée est un débit, une cote ou un volume. C'est de cette manière que l'on introduit la notion bien connue des hydrauliciens (mais mal définie) de commande par l'amont ou de commande par l'aval.

\subsubsection{Commande en boucle fermée}

\subsubsection{Régulation des débits en boucle fermée}

Les organes de commande du système (vannes, seuils) sont manœuvrés en fonction des écarts entre les débits contrôlés en divers points du système et les débits objectifs correspondants.

\section{Exemples de régulation}

- GPC (Sawadogo 1992a),

- CACG (Piquereau et al. 1982),

- CARA (Marzouki 1989, Roux 1992).

\subsubsection{Régulation des cotes en boucle fermée}

Les organes de commande du système sont manœuvrés en fonction des écarts entre les cotes contrôlées en divers points du système et les cotes de consigne correspondantes.

L'écoulement dans les canaux étant généralement de type fluvial, une cote peut être contrôlée, soit par modification du débit amont, soit par modification de la cote aval (tant que la cote contrôlée reste dans la courbe de remous de la cote aval modifiée). Ces modifications se font en agissant (respectivement), soit sur un ouvrage amont (fig. 12), soit sur un ouvrage aval (fig. 13). Paradoxalement, la première logique de contrôle est appelée commande par l'aval, la seconde logique est appelée commande par l'amont.

La commande (en cote) par l'aval conduit indirectement à une commande en débit en boucle fermée, puisqu'elle est obtenue par modification du débit amont. Cette caractéristique est une propriété essentielle de la commande (en cote) par l'aval. En effet, c'est grâce à cette particularité remarquable qu'une commande en cote par l'aval se suffit à elle-même, et qu'aucune boucle de régulation supplémentaire en débit n'est nécessaire (ni en boucle ouverte, ni en boucle fermée).

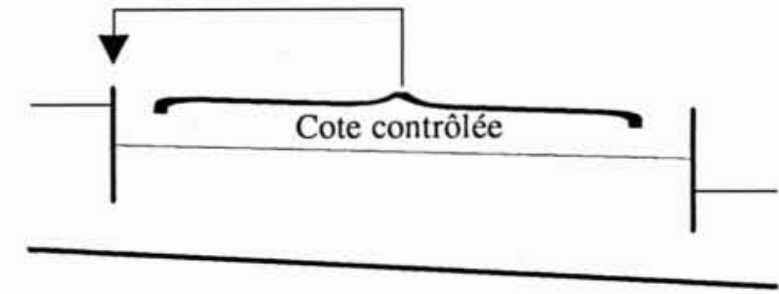

12. Commande par l'aval.

- LittleMan par l'aval (USBR),

- ELFLO (Shand 1971),

- CARDD (Burt 1983),

- Zimbelman (1981),

- CARA (régulation d'une cote dans une bâche, Marzouki 1989),

- BIVAL (SOGREAH),

- PI (Sogreah - Kirkuk, Irak),

- PID (Chevereau 1991),

- PIR (Deltour 1992).

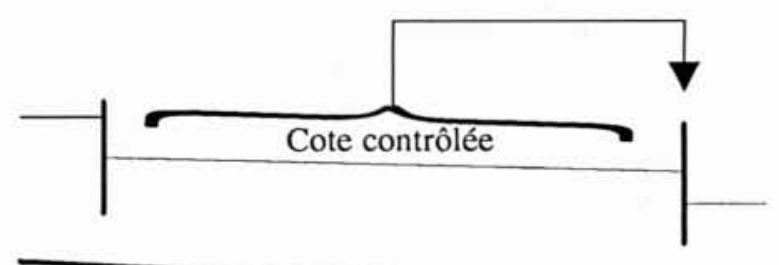

13. Commande par l'amont.

Contrairement à la commande (en cote) par l'aval, la commande (en cote) par l'amont ne conduit pas à une commande en débit, puisqu'elle est obtenue par simple modification de la cote aval. Une commande (en cote) par l'amont ne se suffit donc pas en elle-même et doit être complétée par une commande en débit (en boucle ouverte ou en boucle fermée).

\section{Exemples de régulation}

- Vannes AMIL (Alsthom Fluide),

- LittleMan par l'amont (USBR),

- PID (UMA Engineering - Imperial Valley, USA),

- Seuils bec de canard (même si ces ouvrages sont fixes, ils peuvent être considérés comme fonctionnant en boucle fermée dans la mesure où ils maintiennent une cote quasiconstante quelles que soient les perturbations agissant sur le système).

Certaines commandes en cote combinent les deux logiques, par l'amont et par l'aval (fig. 14). Dans la mesure où elles conduisent également à une commande en débit en boucle fermée elles sont également appelées, pour simplifier, commandes par l'aval. Lorsque l'on veut introduire une terminologie particulière elles sont appelées commandes mixtes. 


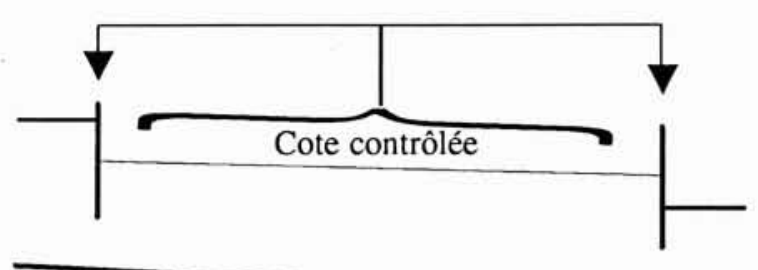

14. Commande mixte.

\section{Exemples de régulation}

- Vannes mixtes (Alsthom Fluide, exploitées par la CNABRL (11)). Le principe de fonctionnement ou de régulation de ces vannes est parfois dit «à niveaux associés ", car, dans certaines gammes de fonctionnement, la vanne a pour but de maintenir une différence constante entre ses cotes amont et aval. Nous classons ces vannes dans la catégorie des vannes en «commande mixte", car la modification d'une cote dans un bief implique la réaction combinée des vannes amont et aval à cette cote.

- LQR (12) (Corriga et al. 1980, 1982a, 1982b et 1983, Balogun 1985 et 1988, Garcia 1988 et 1992, Hubbard et al. 1987, Reddy 1986, 1990, 1992a et 1992b, Filipovic et al. 1989, Florea et al., Malaterre 1994, Sawadogo et al. 1994).

\subsubsection{Régulation des volumes en boucle fermée}

Les organes de commande du système sont manœuvrés en fonction des écarts entre les volumes contrôlés en divers biefs du système et les volumes de consigne correspondants. En général, le volume d'un bief est contrôlé grâce aux manœuvres de la vanne amont de ce bief. De ce fait, la commande en volume est également une commande en débit.

\section{Exemples de régulation}

- Régulation Dynamique (SCP),

- Controlled Volumes (USBR).

\subsubsection{Commande en boucle ouverte}

\subsubsection{Régulation des débits en boucle ouverte}

Les organes de commande du système sont manœuvrés en fonction de la connaissance des débits de consigne, d'une estimation des prélèvements et de la dynamique du système.

\section{Exemples de régulation}

- Par inversion de modèle :

- onde cinématique ou retard pur (CARA, Roux 1992),

- onde diffusive (CACG, Sawadogo 1992a),

- onde dynamique (Chevereau 1991 : SIMBAK, O'Laughlin 1972, Liu et al. 1992, USBR 1979 et Falvey 1987 : Gate Stroking).

(') Compagnie Nationale d'Aménagement du Bas-Rhône Languedoc.

(12) Linear Quadradic Regulator, appelé également commande optimale ou contrôle optimal.
Ces différentes méthodes sont étudiées et comparées par Chevereau (1991).

- Par optimisation (Najim 1981, Sabet et al. 1985, Tomicic 1989, Khaladi 1992, Zihui et al. 1992).

\subsubsection{Régulation des cotes en boucle ouverte}

Les organes de commande du système sont manœuvrés en fonction de la connaissance des cotes de consigne, d'une estimation des prélèvements et de la dynamique du système.

\section{Exemples de régulation}

- Par inversion du modèle de l'onde dynamique (O'Laughlin 1972, Liu et al. 1992, USBR 1979, Falvey 1987); - Par optimisation (Tomicic 1989, Khaladi 1992, Zihui 1992);

- Par simulation (Malaterre 1989, Baume et al. 1993 Kirindi Oya, Sri Lanka).

\subsubsection{Régulation des volumes en boucle ouverte}

Les organes de commande du système sont manœuvrés en fonction de la connaissance des volumes de consigne, d'une estimation des prélèvements et de la dynamique du système.

\section{Exemple de régulation}

- Sabet et al. (1985).

Remarque : certains exemples sont cités à la fois en régulation des débits et en régulation des cotes. Par exemple, le modèle de l'onde dynamique permet de calculer simultanément ces deux types de variables.

\subsubsection{Conclusion}

Une régulation en cote par l'aval ou une régulation en volume (par l'aval) conduisent indirectement à une régulation en débit, comme indiqué aux chapitres 3.3.2.2 et 3.3.2.3. Par contre, dans les autres cas (en particulier régulation en cote par l'amont), deux boucles de contrôle sont nécessaires. Couramment, la régulation en débit est effectuée en boucle ouverte et la régulation en cote est effectuée en boucle fermée. Ceci est lié au fait que les besoins sont exprimés en débit (mais les débits sont difficilement mesurables) et que les cotes sont facilement mesurables. Or, une boucle ouverte utilise des prévisions et une boucle fermée utilise des mesures.

Il ne faut pas confondre, comme certains auteurs le font, les variables régulées avec les logiques de contrôle. En effet, une cote régulée à l'amont d'un ouvrage (fig. 7) n'implique pas forcément une commande par l'amont (fig. 13), comme le montrent les régulateurs ELFLO ou PIR. Par ailleurs, une commande par l'amont (fig. 13) n'est pas synonyme de commande en boucle ouverte (fig. 10), les vannes AMIL en sont un exemple.

\subsection{Méthodes de synthèse}

La démarche de l'automatique implique le passage par 3 étapes :

- la modélisation, ou définition d'un modèle,

- l'analyse, ou étude du comportement du modèle, 
- le contrôle, ou détermination d'une commande $U$ conduisant à une dynamique pré-définie du système régulé, ou minimisant un critère $J$.

Les méthodes d'analyse des systèmes et de synthèse des contrôleurs ont beaucoup évolué, de même que les outils utilisés par les ingénieurs. Les premiers outils de calcul scientifique datent de plus de 3 siècles, mais ce n'est que très récemment que l'informatique les a révolutionnés. Actuellement, environ 15 outils de simulation dynamique sont disponibles sur micro-ordinateur. La $19^{\text {ème }}$ Annual Advanced Control Conference (Août 1993) à l'Université de Purdue, fut entièrement consacrée à ces outils de simulation dynamique (Kompass 1993). Au cours des vingt dernières années, ces progrès ont permis aux recherches et applications en automatique de passer de la régulation analogique monovariable à la régulation numérique multivariable dans l'espace d'état. Dans les chapitres qui suivent, nous présentons différentes méthodes utilisées en régulation des canaux, en distinguant les méthodes monovariables des méthodes multivariables.

\subsubsection{Définition des entrées/sorties}

Nous avons évoqué, au chapitre 3.2 , les variables de contrôle $U$ et les variables contrôlées $Y$ d'un système régulé donné. Les variables $U$ sont des entrées (respectivement des sorties) particulières du système (respectivement du contrôleur). Nous notons (fig. 15):

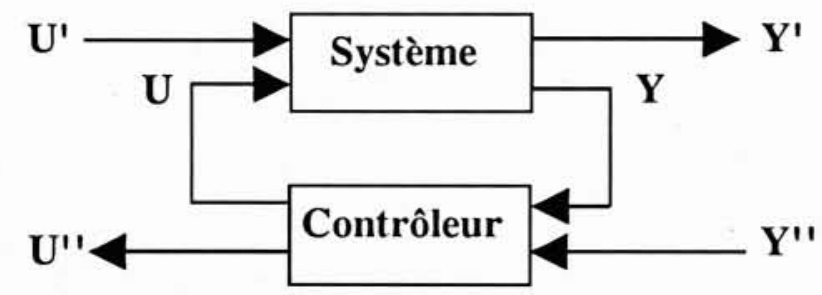

15. Entrées/sorties d'un système.

- $U^{\prime}$ les variables agissant sur le système, mais ne provenant pas du contrôleur considéré (ex. : entrées non commandables, perturbations),

- $U^{\prime \prime}$ les variables générées par le contrôleur, mais n'agissant pas sur le système considéré (ex. : commandes pour un autre système),

- $Y^{\prime}$ les variables générées par le système, mais pas directement contrôlées (ex. : des variables mesurées $Z$ ),

- $Y^{\prime \prime}$ les variables utilisées par le contrôleur, autres que les variables contrôlées $Y$ (ex. : des variables $Z$ mesurées sur le système, des reports de commande U provenant d'autres contrôleurs, etc.).

Par convention, nous choisissons de définir les termes « entrées » et "sorties » en référence au contrôleur et non pas en référence au système, car notre classification concerne les contrôleurs et non pas les systèmes. Les entrées du contrôleur (respectivement les sorties) sont donc les variables notées $Y$ et $Y^{\prime \prime}$ ci-dessus (respectivement $U$ et $\left.U^{\prime \prime}\right)$.

Un contrôleur est dit monovariable (en entrée et en sortie) s'il ne considère qu'une entrée et qu'une sortie (les variables étant entendues au sens scalaire et non pas au sens vectoriel). Les méthodes monovariables sont parfois appelées SISO pour «Single Input, Single Output».

Un contrôleur est dit multivariable (en entrée et en sortie) s'il considère plusieurs entrées et plusieurs sorties (les variables étant entendues au sens scalaire et non pas au sens vectoriel). Les méthodes multivariables sont parfois appelées MIMO pour « Multiple Input, Multiple Output ».

Il existe bien entendu des contrôleurs multivariables uniquement en entrée (MISO) ou uniquement en sortie (SIMO). On peut également indiquer le nombre $n$ d'entrées et le nombre $m$ de sorties du contrôleur en qualifiant la méthode de $n I m O$ (ex. : $3 I$ 2O).

Dans le cas d'un report de commande d'un contrôleur vers les contrôleurs adjacents amont ou aval (Régulation Dynamique, PIR ou découpleurs d'ELFLO), on considère ce report comme une entrée supplémentaire du contrôleur, mais pas comme une sortie supplémentaire.

Par abus de langage, on appelle souvent «monovariable » une méthode ayant une sortie unique, même si elle considère plusieurs entrées $(n I 1 O)$, et "multivariable », une méthode à plusieurs sorties $(n I m O, m>1)$. C'est en effet le nombre de sorties générées par le contrôleur qui conditionnent directement ses fondements théoriques et mathématiques. Cet abus de langage sous-tend les deux chapitres qui suivent.

\subsubsection{Méthodes monovariables}

Des méthodes heuristiques monovariables ont été développées à partir de considérations hydrauliques. Bien que citées dans la littérature elles sont, à notre connaissance, peu opérationnelles et difficilement transposables d'un site à un autre :

- Zimbelman (1987),

- CARDD (Burt 1983).

Une méthode empirique utilisée par la CARA sur des systèmes rivières-bâches (Marzouki 1989) est en cours de modernisation (Roux 1992).

Une autre méthode empirique basée sur un contrôleur à 3 positions a été développée aux USA :

- LittleMan (USBR, Buyalski 1991).

La plupart des autres méthodes de régulation développées et testées sur les canaux d'irrigation sont basées sur des contrôleurs linéaires monovariables en boucle fermée de type PID (Proportionnel, Intégral, Dérivé) :

- P (Proportionnel) :

- AMIL, AVIS, AVIO (Alsthom Fluide).

- PI (Proportionnel, Intégral) :

- ELFLO (Shand 1971),

- BIVAL (SOGREAH),

- Régulation Dynamique (SCP),

- PI (SOGREAH - Kirkuk, Irak).

- PID (Proportionnel, Intégral, Dérivé) :

- PID (UMA Engineering - Imperial Valley, USA).

- Régulateur linéaire d'ordre supérieur à 2 , calé par placement de pôle : 


\section{- CACG.}

Bien que très performantes dans la plupart des cas, ces méthodes présentent l'inconvénient de ne pas prendre en compte explicitement les temps de retard caractéristiques des biefs de canaux. Pour y remédier, Shand (1971) a étudié la possibilité d'utiliser un Prédicteur de Smith pour automatiser le Corning Canal, Californie, USA. Cependant, la réalisation d'un opérateur retard analogique posait des problèmes technologiques à l'époque. De ce fait, et malgré des performances moindres, la méthode ELFLO a été finalement retenue.

Récemment, l'idée d'utiliser un Prédicteur de Smith, combiné avec un contrôleur PI, a été reprise et détaillée (Deltour 1992, Sanfilippo 1993). L'utilisation de la technologie numérique a maintenant supprimé les problèmes rencontrés par Shand. Ce contrôleur a été appelé PIR (Proportionnel, Intégral, Retard).

Les coefficients des régulateurs de type PID sont ajustés soit par tâtonnement, soit par la méthode de ZieglerNichols (Larminat 1993, Aström et al. 1993), soit par placement de pôle.

Récemment, des méthodes basées sur la commande floue (ex. : CNABRL sur le canal du T2 à Marrakech, Maroc), les systèmes experts ou les réseaux de neurones (ANN de Schaalje et Manz, et Thèse de Doctorat en cours au CEMAGREF) ont été développées. Ces méthodes sont encore à l'état de recherche.

Les méthodes monovariables présentent l'inconvénient de nécessiter un découpage du système en sous-systèmes sans tenir compte explicitement des interactions entre ces sous-systèmes. Or, un canal d'irrigation est typiquement un système multivariable présentant de fortes interactions entre sous-systèmes (la manœuvre d'une vanne a des influences sur plusieurs biefs amont et aval). La technique du découplage a pour objectif de limiter, dans la mesure du possible, l'influence d'une commande sur la seule sortie contrôlée par le régulateur. Le processus global multivariable peut alors être considéré comme une série de processus indépendants monovariables non interactifs évoluant en parallèle. Ceci est possible si le nombre d'entrées est supérieur ou égal au nombre de sorties (Borne et al. 1990). Les performances d'un contrôleur peuvent être notablement améliorées par découplage. Cette technique n'a cependant été appliquée qu'une seule fois pour le contrôleur ELFLO (Schuurmans 1992). Ce découplage est réalisé à partir d'un modèle du processus. Ses performances sont donc dégradées du fait des perturbations inconnues et des erreurs de modèle (non linéarités). D'autres méthodes (ex. : PIR de Deltour 1992, Régulation Dynamique SCP), bien que ne tenant pas compte explicitement des problèmes de couplage, tentent de les limiter en considérant le débit et non l'ouverture comme variable de contrôle à un ouvrage (équivalent du découpleur II de Schuurmans 1992) et en reportant une partie d'une commande à un ouvrage sur l'ouvrage situé à son amont (équivalent du découpleur I de Schuurmans 1992).

\subsubsection{Méthodes multivariables}

Les automaticiens utilisent des méthodes multivariables basées sur des placements de pôle dans l'espace d'état, ou sur des PID multivariables. Cependant aucune de ces méthodes n'a été utilisée sur les canaux, à notre connaissance.
Des méthodes par inversion de modèle sont décrites dans la littérature, conduisant généralement à des boucles ouvertes (Chevereau 1991, Liu et al. 1992), et éventuellement à des boucles fermées (Liu et al. 1994).

Des méthodes d'optimisation ont été développées par différents auteurs. Elles sont basées non plus sur la réponse désirée en boucle fermée, mais sur la minimisation d'un critère $J$ pondérant la commande et les écarts entre les variables contrôlées et leurs consignes. Ces méthodes présentent l'avantage d'être naturellement multivariables : - Optimisation linéaire (Sabet 1985),

- Optimisation non linéaire (Tomicic 1989, Khaladi 1992, Zihui 1992),

- GPC (Sawadogo 1992, Rodellar 1993),

- LQR (Corriga 1983, Balogun 1985, Garcia 1988, Hubbard 1987, Reddy 1992, Filipovic 1989, Florea, Malaterre 1994, Sawadogo et al. 1994).

Remarque : la méthode GPC a été utilisée par Sawadogo (1992) sous forme monovariable, mais elle est citée ici, dans le chapitre des méthodes multivariables, car elle en a le potentiel.

La mise en œuvre de ces méthodes est beaucoup plus lourde que dans le cas monovariable. L'optimisation nonlinéaire classique conduit uniquement à une boucle ouverte, sensible aux erreurs et aux perturbations. Pour introduire une boucle fermée, l'optimisation doit être refaite périodiquement (voire à chaque pas de temps), ce qui alourdit la méthode et limite ses applications (problèmes de temps réel). Par ailleurs, la détermination des conditions initiales réelles, nécessaires à l'optimisation, n'est a priori pas évidente.

Les méthodes GPC (modèle sous forme de fonctions de transfert, Chan et al. 1990, Soeterboek 1990, Lee et al. 1990, Linkens et al. 1992) et LQR (modèle dans l'espace d'état) présentent l'avantage d'incorporer naturellement une boucle ouverte et une boucle fermée.

\subsection{Mises en æuvre}

Différentes mises en œuvre sont possibles pour chaque méthode de régulation. Les plus courantes sont décrites dans la littérature (Plusquellec 1988, Goussard 1993, Plusquellec et al. 1994, p. 36). Nous les listerons dans ce chapitre, sans les décrire en détail, par soucis de concision.

Remarque: il ne faut pas confondre la "méthode de synthèse ", présentée dans le chapitre précédent, avec la "mise en auvre». Ainsi un régulateur provenant d'une méthode de synthèse donnée (ex. : un contrôleur de type PI) peut être aussi bien piloté localement, i.e. individuellement bief par bief (ex. : ELFLO), qu'à distance, à partir d'un poste de contrôle centralisé (ex. : Régulation Dynamique de la $S(P)$. De même, un ouvrage de régulation (manuel ou motorisé) donné peut être aussi bien manœuvré sur place par un opérateur humain (BIVAL à l'Office du Niger, Mali), que commandé automatiquement par un automate programmable numérique (BIVAL usuel, par exemple au Rio Cupatitzio-Tepalcatepec, Mexique).

Dans la mise en œuvre d'une méthode de régulation, différents aspects peuvent être distingués, en particulier: - l'architecture (localisée, centralisée, etc.),

- les ouvrages (seuils fixes, vannes manuelles, vannes automatiques, etc.), 
- l'instrumentation (type de capteur pour des mesures de cote : piézo-résistif, ultrason immergé, ultrason sec, bulleà-bulle, flotteur, visuel, etc. ou pour des mesures de débit : moulinet, magnétique, laser, etc.),

- les communications (messager, ligne de transmission PTT, ligne privée, radio-transmission, etc.),

- les calculs et traitements de l'information (méthode empirique, abaque, règle à calcul, principe hydromécanique, machine à calculer, micro-ordinateur, station de travail, gros systèmes, etc.).

Nous nous limiterons, dans les chapitres suivants, aux aspects " architecture » et «ouvrages », qui nous paraissent les plus essentiels pour la compréhension du fonctionnement et des caractéristiques (avantages et inconvénients, contraintes) d'une méthode de régulation.

\subsubsection{Architectures}

Les différentes architectures de mise en œuvre d'une régulation peuvent être classées dans l'ordre croissant de complexité suivant :

- locale proche (fig. 16) : chaque ouvrage est commandé par un contrôleur indépendant, qui n'utilise que des informations locales (variables mesurées), situées à proximité (quelques mètres maximum) de l'ouvrage (ex. : PID UMA Engineering, LittleMan, Vannes Alsthom Fluide).

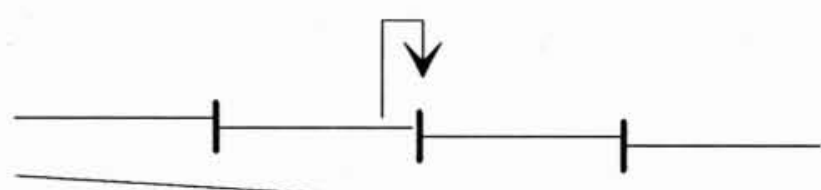

16. Architecture locale proche.

- locale distante (fig. 17): idem ci-dessus, mais les variables mesurées peuvent être distantes de l'ouvrage, par exemple situées à l'extrémité aval du bief (ex. : ELFLO, PIR, BIVAL, CARDD, Zimbelman).

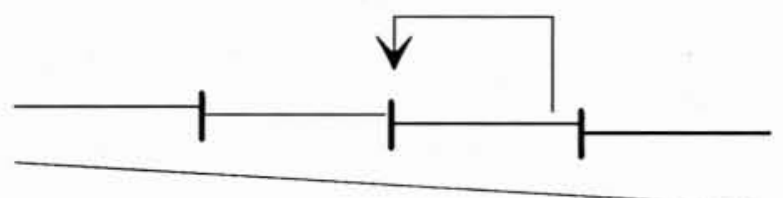

\section{Architecture locale distante.}

- semi-locale (fig. 18) : idem ci-dessus, mais les contrôleurs ne sont plus indépendants, et utilisent explicitement des données générées par les contrôleurs adjacents, par exemple un report d'ouverture du contrôleur aval vers le contrôleur amont (ex. : PIR, ELFLO + découpleur).

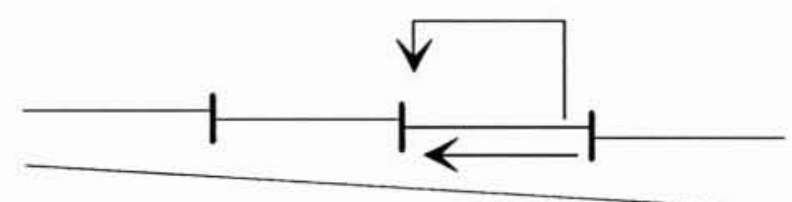

\section{Architecture semi locale.}

- centralisée (fig. 19) : toutes les commandes proviennent d'un contrôleur central (qu'il soit humain ou informatique), éloigné des différents ouvrages. Les commandes peuvent être données en terme d'ouverture (ex. : Salt River Project), en débit ou en cote (ex. : Central Arizona Project, Maricopa Stanfield Irrigation and Drainage District). Une commande centralisée permet la surveillance et la télégestion du réseau. Cependant, elle est plus lourde à mettre en œuvre et plus sensible aux pannes matérielles que des architectures localisées.

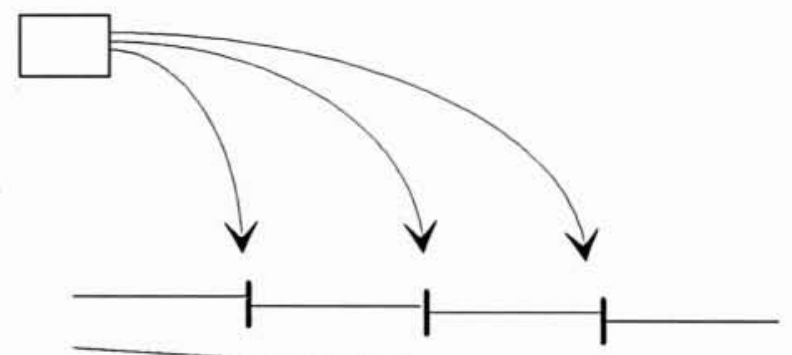

\section{Architecture centralisée.}

- hiérarchisée (fig. 20) : la commande d'un ouvrage dépend de différents niveaux hiérarchisés de prise de décision. Par exemple, des ouvrages automatiques à contrôleurs locaux distants, communiquent avec un poste de contrôle central (modification des consignes, surveillance des pannes, etc.).

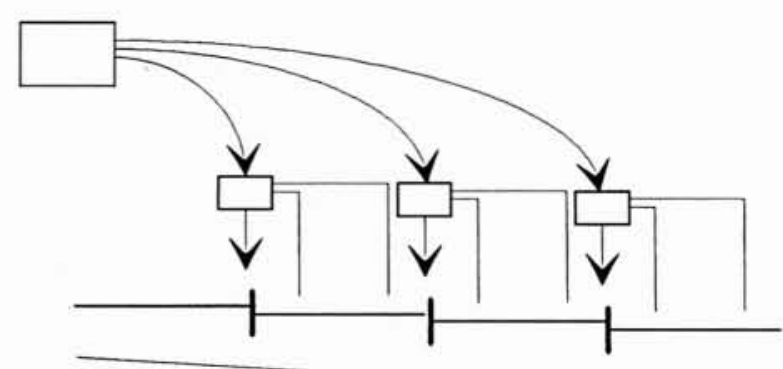

20. Architecture hiérarchisée. 
Remarque : potentiellement, la composante «boucle fermée " de la Régulation Dynamique pourrait être semilocale. Cependant, la mise en auvre de cette régulation au Canal de Provence est centralisée. Ceci permet de considérer la boucle ouverte et la boucle fermée au même niveau de contrôle, et de superviser le système. Une commande hiérarchisée semblerait bien adaptée à ce type de régulation.

\subsubsection{Ouvrages}

Les différents types d'ouvrage, permettant la mise en œuvre d'une méthode de régulation, peuvent être classés dans l'ordre croissant de complexité suivant (un ouvrage peut être une vanne ou éventuellement un seuil) :

- ouvrage fixe (ex. : seuil bec de canard, partiteur proportionnel, etc.),

- ouvrage manuel,

- ouvrage motorisé,

- ouvrage automatique mécanique à flotteur (vannes AMIL, AVIS, AVIO, etc.),

- ouvrage automatique électronique ou électromécanique.

Les vannes mécaniques à flotteurs présentent l'avantage d'être relativement bon marché, suffisamment précises et de maintenance facile. Elles sont très bien adaptées aux canaux de petite ou de moyenne taille. Les vannes électroniques ou électromécaniques présentent les avantages d'être plus précises et plus souples d'utilisation (possibilité de changer les consignes ou algorithmes de fonctionnement, possibilité de les commander à distance, etc.). Elles sont cependant plus coûteuses, moins fiables, et de maintenance plus exigeante. Elles sont de ce fait réservées aux canaux de grande taille.

\section{CONCLUSION}

Après une présentation du contexte général de l'irrigation dans le monde, nous avons montré en quoi les classifications des méthodes de régulation des canaux trouvées dans la littérature manquent de structure et de rigueur.
Nous avons tenté de combler cette lacune en définissant et illustrant les quatre critères essentiels de caractérisation d'une méthode de régulation: les variables considérées (contrôlées, mesurées, et de contrôle), les logiques de contrôle, les méthodes de synthèse (architectures et techniques) et les mises en œuvre (architectures et ouvrages).

Dans le tableau 1, nous décrivons toutes les méthodes citées dans la littérature, à travers cette caractérisation. Certaines données sont cependant manquantes ou sujettes à caution, du fait du manque de clarté de la littérature disponible. Des informations autres que celles relatives aux quatre critères de base ont également été indiquées dans le tableau. En particulier, le nom des auteurs des méthodes, et des exemples d'application ou de test ont été donnés. Les abréviations utilisées dans le tableau sont explicitées en fin d'article. Le signe «\& » signifie « et », «- " signifie néant ou sans objet, «? " signifie inconnu de l'auteur, ou sujet à caution. Le tableau aurait pu être classé selon un des quatre critères de caractérisation. Cependant, afin de ne pas privilégier un critère par rapport aux autres, nous avons présenté le tableau dans l'ordre alphabétique des noms des méthodes.

Une fois ce travail de caractérisation terminé, la classification proprement dite peut être réalisée facilement. Par exemple, on peut décider de classifier les méthodes en fonction de leur logique de contrôle. On peut créer la partition suivante, à trois parties : $B O$ seule, $B F$ seule, $B O$ $+B F$. On peut raffiner la partition, si nécessaire, en subdivisant ces parties par l'introduction des notions de $B F_{a m}$, $B F_{m i}$ et $B F_{a r}$

On peut définir une nouvelle classification en fonction de l'architecture de mise en $\propto u v r e$, en définissant la partition: locale proche, locale distante, semi-locale, centralisée, hiérarchisée.

L'avantage d'effectuer de telles classifications est d'avoir une vision plus claire des caractéristiques de chaque méthode, et ainsi de ses propriétés. En effet, à chaque caractéristique correspondent directement des avantages, des inconvénients et des contraintes. A l'inverse, si l'on se fixe des propriétés souhaitées pour une méthode de régulation donnée, on peut retrouver la ou les méthodes satisfaisant ces critères, s'il en existe.

Tableau 1. - Classification des méthodes de régulation.

\begin{tabular}{|c|c|c|c|c|c|c|c|c|c|c|}
\hline \multicolumn{2}{|c|}{ IDENTIFICATION } & \multicolumn{6}{|c|}{ DESCRIPTION } & \multicolumn{3}{|c|}{ APPLICATION } \\
\hline \multirow{2}{*}{$\begin{array}{l}\text { Nom de la méthode } \\
\text { de régulation }\end{array}$} & \multirow{2}{*}{ Auteur(s) } & \multicolumn{3}{|c|}{ Les variables considérées } & \multirow{2}{*}{$\begin{array}{l}\text { Logique } \\
\text { de } \\
\text { contrôle }\end{array}$} & \multicolumn{2}{|c|}{ Méthode de synthèse } & \multicolumn{2}{|c|}{ Mise en œuvre } & \multirow{2}{*}{$\begin{array}{l}\text { Réalisations } \\
\text { ou tests }\end{array}$} \\
\hline & & contrölèes & mesurées & $\begin{array}{c}\text { de } \\
\text { contrôle }\end{array}$ & & $\begin{array}{l}\text { Archi- } \\
\text { tecture }\end{array}$ & Technique & Architecture & Ouvrages & \\
\hline ACS & CAP, USBR & $0 \& y$ & - & $w$ & $B O$ & MIMO & $\begin{array}{l}\text { Inversion de mo- } \\
\text { dele }\end{array}$ & Centralisée & $\begin{array}{l}\text { Vannes auto. } \\
\text { électroniques + } \\
\text { ordinateur }\end{array}$ & $\begin{array}{l}\text { Central Arizona Pro. } \\
\text { ject }\end{array}$ \\
\hline AMIL. & Alsthom Fluide & $y_{a v}$ & $y_{a v}$ & $w$ & $B F_{a m}$ & siso & P & Locale proche & $\begin{array}{l}\text { Vannes auto. } \\
\text { mécaniques }\end{array}$ & Nombreux pays \\
\hline ANN & Schaalje \& Manz & $y$ & y & $w$ & $B F_{m i}$ & MIMO & $\begin{array}{l}\text { Réseaux de neu- } \\
\text { rones }\end{array}$ & Centralisée & $\begin{array}{l}\text { Vannes auto. } \\
\text { électroniques + } \\
\text { ordinateur }\end{array}$ & - \\
\hline AVIS, AVIO & Alsthom Fluide & $y_{a m}$ & $y_{a m}$ & $w$ & $B F_{a v}$ & siso & $P$ & Locale proche & $\begin{array}{l}\text { Vannes auto. } \\
\text { mécaniques }\end{array}$ & Nombreux pays \\
\hline BIVAL & SOGREAH & $\begin{array}{l}\text { a. } y_{a m}+ \\
(1-a) \cdot y_{a v}\end{array}$ & $y_{a m} \& y_{a v}$ & $w$ & $B F_{a v}$ & siso & PI & Locale distante & $\begin{array}{l}\text { Vannes auto. } \\
\text { èlectroniques }\end{array}$ & Nombreux pays \\
\hline CARA & CARA & $\begin{array}{l}\text { Q \& y } \\
\text { báche }\end{array}$ & $\begin{array}{l}\text { Q \& y } \\
\text { báche }\end{array}$ & $Q$ & $B F_{a v}+B O$ & SISO & Heuristique & Locale distante & $\begin{array}{l}\text { Vannes auto. } \\
\text { électroniques }\end{array}$ & $\begin{array}{l}\text { Rivières du Sud } \\
\text { Ouest de la France }\end{array}$ \\
\hline CARAMBA & $\begin{array}{l}\text { CACG - CEMA- } \\
\text { GREF }\end{array}$ & $Q_{a v}$ & $\begin{array}{l}Q_{a y} \& \\
2 Q_{i n}\end{array}$ & $Q_{a m}$ & $B F+B O$ & 3ISO & $\begin{array}{l}\text { Placement de } \\
\text { pôles }\end{array}$ & Centralisée & $\begin{array}{l}\text { Vannes auto. } \\
\text { électroniques }\end{array}$ & $\begin{array}{l}\text { Rivieres du Sud } \\
\text { Ouest de la France }\end{array}$ \\
\hline
\end{tabular}




\begin{tabular}{|c|c|c|c|c|c|c|c|c|c|c|}
\hline \multicolumn{2}{|c|}{ IDENTIFICATION } & \multicolumn{6}{|c|}{ DESCRIPTION } & \multicolumn{3}{|c|}{ APPLICATION } \\
\hline \multirow{2}{*}{$\begin{array}{l}\text { Nom de la méthode } \\
\text { de régulation }\end{array}$} & \multirow{2}{*}{ Auteur(s) } & \multicolumn{3}{|c|}{ Les variables considérées } & \multirow{2}{*}{$\begin{array}{l}\text { Logique } \\
\text { de } \\
\text { contrōle }\end{array}$} & \multicolumn{2}{|c|}{ Méthode de synthèse } & \multicolumn{2}{|c|}{ Mise en œuvre } & \multirow{2}{*}{$\begin{array}{l}\text { Réalisations } \\
\text { ou tests }\end{array}$} \\
\hline & & contrólées & mesurées & $\begin{array}{c}\text { de } \\
\text { contróle }\end{array}$ & & $\begin{array}{l}\text { Archi- } \\
\text { tecture }\end{array}$ & Technique & Architecture & Ouvrages & \\
\hline CARDD & $\begin{array}{l}\text { Charles Burt \& } \\
\text { Parrish }\end{array}$ & $y_{a v}$ & $3-5 y_{\text {in }}$ & $w$ & $B F_{a v}$ & 3.5150 & Heuristique & Locale distante & $\begin{array}{l}\text { Vannes auto. } \\
\text { électroniques }\end{array}$ & $\begin{array}{l}\text { Canal réduit de Cal- } \\
\text { Poly }\end{array}$ \\
\hline CEMAGREF & CEMAGREF & $y_{a v}$ & $y_{a v}$ & $w$ & $\begin{array}{l}B F_{a m}+ \\
B O\end{array}$ & MIMO & $\begin{array}{l}\text { Simulation sur } \\
\text { modèle hydrauli- } \\
\text { que }\end{array}$ & Centralisée & $\begin{array}{l}\text { Vannes manuel- } \\
\text { les }\end{array}$ & Kirindi Oya \\
\hline CEMAGREF & $\begin{array}{l}\text { CEMAGREF (Ma- } \\
\text { laterre, Kosuth) }\end{array}$ & $\begin{array}{l}\text { Q \& y } \\
\text { tres } \\
\text { souple }\end{array}$ & $\begin{array}{l}y_{a m} \gamma_{a y} \\
\text { event }\end{array}$ & $O \& w$ & $B F_{m i}+B O$ & MIMO & $\begin{array}{l}\text { LQR + observa. } \\
\text { teur réduit }\end{array}$ & Centralisée & $\begin{array}{l}\text { Vannes auto. } \\
\text { électroniques + } \\
\text { ordinateur }\end{array}$ & Modèle non linéaire \\
\hline Chevereau & Chevereau & $y_{a v}$ & $y_{a v}$ & $w$ & $B F$ & SISO & PID & Locale distante & $\begin{array}{l}\text { Vannes auto. } \\
\text { électroniques }\end{array}$ & Modèle non linéaire \\
\hline $\begin{array}{l}\text { Controlled Volu- } \\
\text { mes }\end{array}$ & USBR & $v$ & $?$ & $w$ & $B F_{a v}+B O$ & MIMO & $?$ & Centralisée & $\begin{array}{l}\text { Vannes auto. } \\
\text { électroniques }\end{array}$ & $\begin{array}{l}\text { California } \\
\text { Aqueduc }\end{array}$ \\
\hline DACL & & $?$ & $?$ & $?$ & $B F$ & SISO & $?$ & Locale proche & $?$ & $?$ \\
\hline ELFLO & $\begin{array}{l}\text { Shand (Berkeley } \\
\text { U.). Buyalski \& } \\
\text { Serfozo }\end{array}$ & $y_{a v}$ & $y_{a v}$ & $w$ & $B F_{a v}$ & SISO & $\mathrm{PI}+$ Filtre & Locale distante & $\begin{array}{l}\text { Vannes auto. } \\
\text { électromécani- } \\
\text { ques ou électro- } \\
\text { niques }\end{array}$ & $\begin{array}{l}\text { Corning, Coalinga } \\
\text { (USA) }\end{array}$ \\
\hline $\begin{array}{l}\text { ELFLLO + Décou- } \\
\text { pleur }\end{array}$ & Schuurmans & $y_{a v}$ & $y_{a v} \& w$ & $w$ & $B F_{a v}$ & $2-3150$ & $\begin{array}{l}\mathrm{PI}+\text { Filtre + dé- } \\
\text { coupleur }\end{array}$ & $\begin{array}{l}\text { Semi-locale, } \\
\text { distante }\end{array}$ & $\begin{array}{l}\text { Vannes auto. } \\
\text { electroniques + } \\
\text { ordinateur }\end{array}$ & $\begin{array}{l}\text { Canal réduit de Cal- } \\
\text { Poly }\end{array}$ \\
\hline Gate Stroking & Wylie, Falvey & $Q \& y_{a v}$ & - & $w$ & BO & MIMO & $\begin{array}{l}\text { Inversion de mo- } \\
\text { dèle }\end{array}$ & Centralisée & $\begin{array}{l}\text { Vannes auto. } \\
\text { électroniques }\end{array}$ & $\begin{array}{l}\text { Granite Reef Aque- } \\
\text { duc (CAP) }\end{array}$ \\
\hline $\begin{array}{l}\text { IMTA - CEMA- } \\
\text { GREF }\end{array}$ & $\begin{array}{l}\text { IMTA - CEMA- } \\
\text { GREF }\end{array}$ & $y_{a v}$ & $y_{a v}$ & $a$ & $B F_{a v}+B O$ & Siso & $\mathrm{PI}$ & Locale distante & $\begin{array}{l}\text { Vannes auto. } \\
\text { électroniques }\end{array}$ & Begonia \\
\hline Khaladi & $\begin{array}{l}\text { CEMAGREF } \\
\text { (Ahmed Khaladi) }\end{array}$ & $Q \& y$ & $?$ & Q\& $W$ & $\begin{array}{l}B O(+ \\
B F ?)^{+}\end{array}$ & MIMO & $\begin{array}{l}\text { Optimisation non- } \\
\text { linéaire }\end{array}$ & Centralisée & $\begin{array}{l}\text { Vannes auto. } \\
\text { électroniques + } \\
\text { ordinateur }\end{array}$ & Modèle non linéaire \\
\hline $\begin{array}{l}\text { LittleMan (par } \\
\text { l'amont) }\end{array}$ & & $y_{a v}$ & $y_{a v}$ & $w$ & $B F_{a m}$ & SISO & 3 positions & Locale proche & $\begin{array}{l}\text { Vannes auto. } \\
\text { électromécani- } \\
\text { ques }\end{array}$ & $?$ \\
\hline $\begin{array}{l}\text { LittleMan (par } \\
\text { l'aval) }\end{array}$ & & $y_{a m}$ & $y_{a m}$ & $w$ & $B F_{a v}$ & Siso & 3 positions & locale proche & $\begin{array}{l}\text { Vannes auto. } \\
\text { electromécani- } \\
\text { ques }\end{array}$ & $?$ \\
\hline Liu & Liu et al. & $Q \& y_{a v}$ & $?$ & $w$ & $\begin{array}{l}\mathrm{BO}(+ \\
B F ?)\end{array}$ & MIMO & $\begin{array}{l}\text { Inversion de mo. } \\
\text { dèle }\end{array}$ & Centralisée & & Modèle \\
\hline LQR Davis U. & Balogun, Garcia & $Q \& y$ & $y$ & $Q \& w$ & $B F_{m i}$ & MIMO & LQR & Centralisée & $\begin{array}{l}\text { Vannes auto. } \\
\text { électroniques + } \\
\text { ordinateur }\end{array}$ & Modèle \\
\hline $\begin{array}{l}\text { Model Predictive } \\
\text { Control }\end{array}$ & Zagona \& Clough & $Q \& y$ & $?$ & $w$ & $B F+B O$ & MIMO & $\begin{array}{l}\text { Commande pré- } \\
\text { dictive }\end{array}$ & Centralisée & & \\
\hline Najim & Najim & $y$ & $y$ & $Q$ & $B F+B O$ & $?$ & $\begin{array}{l}\text { Automate à struc- } \\
\text { ture variable }\end{array}$ & Centralisée & & \\
\hline NLP & Lin \& Manz & $?$ & $?$ & $?$ & $B F+B O$ & $?$ & $\begin{array}{l}\text { Optimisation non } \\
\text { linéaire }\end{array}$ & Centralisée & . & \\
\hline O'Laughlin & O'Laughlin & $Q \& y_{a v}$ & - & $Q \& w$ & $B O$ & MIMO & $\begin{array}{l}\text { Inversion de mo- } \\
\text { dèle }\end{array}$ & Centralisée & & \\
\hline$P+P R$ & Buyalski & $y_{a m}$ & $y_{a m}$ & $w$ & $B F_{a v}$ & SISO & $\mathrm{PI}+$ Filtre & Locale proche & $\begin{array}{l}\text { Vannes électro- } \\
\text { mécaniques ou } \\
\text { électroniques }\end{array}$ & \\
\hline PIR & SCP (Deltour) & $y_{a v}$ & $y_{a v}$ & $Q$ & $\begin{array}{l}B F_{a v}+ \\
B O^{-}\end{array}$ & 21so & $\begin{array}{l}\mathrm{PI}+\text { Prédicteur } \\
\text { de Smith }\end{array}$ & $\begin{array}{l}\text { Locale distante, } \\
\text { ou semi locale }\end{array}$ & $\begin{array}{l}\text { Vannes auto. } \\
\text { électroniques }\end{array}$ & France (SCP) \\
\hline Reddy 1 & Reddy & $y$ & $y$ & $w$ & $B F_{m i}$ & MIMO & LQR & Centralisée & $\begin{array}{l}\text { Vannes auto. } \\
\text { électroniques + } \\
\text { ordinateur }\end{array}$ & Modèle \\
\hline $\begin{array}{l}\text { Règulation Dyna- } \\
\text { mique }\end{array}$ & SCP & $v$ & $\begin{array}{l}V, y_{a m} \& \\
y_{a v}\end{array}$ & $Q$ & $\begin{array}{l}B F_{a v}+ \\
B O\end{array}$ & 2Iso & $\mathrm{PI}+$ Heuristique & Centralisée & $\begin{array}{l}\text { Vannes auto. } \\
\text { électroniques }\end{array}$ & France, Maroc \\
\hline RTUQ & Rogers & $Q$ & $?$ & $w$ & $B F$ & siso & Heuristique & Locale proche & $\begin{array}{l}\text { Vanne auto. élec- } \\
\text { tronique }\end{array}$ & \\
\hline Sabet & Sabet et al. & $v$ & - & $Q$ & $B O$ & MIMO & $\begin{array}{l}\text { Optimisation } \\
\text { linéaire }\end{array}$ & Centralisèe & & \\
\hline Sawadogo & Sawadogo & $Q$ & $Q$ & $Q$ & $B F+B O$ & siso & GPC & Locale distante & $\begin{array}{l}\text { Vannes auto. } \\
\text { électroniques }\end{array}$ & Modèle non linéaire \\
\hline $\begin{array}{l}\text { Seuils Bec de ca- } \\
\text { nard }\end{array}$ & & $y_{a v}$ & - & - & $B F_{a m}$ & siso & Dimensionnement & Locale proche & Seuil fixe & Nombreux pays \\
\hline SIMBAK & Chevereau & $Q$ & - & $Q$ & $B O$ & siso & $\begin{array}{l}\text { Inversion de mo- } \\
\text { dèle }\end{array}$ & Centralisée & $\begin{array}{l}\text { Vannes auto. } \\
\text { électroniques + } \\
\text { ordinateur }\end{array}$ & Modèle non linéaire \\
\hline Sogreah & Sogreah & $y_{a v}$ & $y_{a v}$ & $w$ & $\begin{array}{l}B F_{a v}+ \\
B O\end{array}$ & siso & $\mathrm{PI}+$ Filtre & Locale distante & $\begin{array}{l}\text { Vannes auto. } \\
\text { électroniques }\end{array}$ & Kirkuk (Irak) \\
\hline Tomicic & Tomicic & $Q \& y$ & - & $Q \& y$ & $\begin{array}{l}B O(+ \\
B F ?)\end{array}$ & MIMO & $\begin{array}{l}\text { Optimisation non } \\
\text { linéaire }\end{array}$ & Centralisée & & Modèle \\
\hline UMA Engineering & UMA Engineering & $y_{a v}$ & $y_{a v}$ & $w$ & $B F_{a m}$ & siso & PID & Locale proche & $\begin{array}{l}\text { Vannes auto. } \\
\text { électroniques }\end{array}$ & Imperial Valley \\
\hline Vannes Mixtes & Alsthom Fluide & $\begin{array}{l}g\left(y_{a m}\right. \\
\left.y_{a v}\right)\end{array}$ & $y_{a m} \& y_{a v}$ & $w$ & $B F_{m i}$ & 2110 & $\mathrm{P}$ ou PI & Locale proche & $\begin{array}{l}\text { Vannes auto. } \\
\text { mécaniques }\end{array}$ & Nombreux pays \\
\hline Zihui & Zihui et al. & $Q \& y$ & - & $Q \& w$ & $\begin{array}{l}B O(+ \\
B F ?)\end{array}$ & $?$ & $\begin{array}{l}\text { Optimisation non } \\
\text { linéaire }\end{array}$ & Centralisée & & Modèle \\
\hline Zimbelman & Zimbelman & $y_{a v}$ & $y_{a v}$ & $w$ & $B F_{a v}$ & siso & Heuristique & Locale distante & $\begin{array}{l}\text { Vannes auto. } \\
\text { électroniques }\end{array}$ & Modèle \\
\hline
\end{tabular}




\section{Références bibliographiques}

AlsTHOM FluIDE, 1975-1979, — « Notice de montage, réglage et entretien des vannes AMIL, AVIS, AVIO \%, 100 p.

ALSTHOM FLUIDE, 1981. - * Gestion de canaux et irrigation, Les matériels NEYRTEC d'équipement pour réseaux régulés », $40 \mathrm{p}$.

ANKUM P., 1992. - - Classification of flow control systems for irrigation $»$, Colloque «Advances in Planniag. Design and Management of Irrigation Systems as Related to Sustainable Land Use », Louvain, 14-17 septembre 1992, édité par Feyen et al., pp. 265-274.

ANKUM P., 1993a. - "Canal storage and flow control methods in irrigation ». Proceedings XVème Congrès ICID, La Haye, Q. 44, R. 51, p 663-679.

ANKUM P., 1993b. - "Self-management in irrigation systems, Proceedings Asian Regional Symposium Beijing, HR Wallingford, édité par Wooldridge et al., session 2, pp. 51-64.

ASCE, 1993. - « Unsteady-flow modeling of irrigation canals ». ASCE Task Committee on irrigation canal system hydraulic modeling, Journal of Irrigation and Drainage Engineering, Vol. $119, \mathrm{n}^{\circ} 4$, pp. 615-630.

Aströ̀ K.J., HÄGglund T., 1993. - « PID Control $»$ à paraître.

Balogun O.S., Hubbard M., De Vries J.J., 1988. - « Automatic control of canal flow using linear quadratic regulator theory », Journal of Hydraulic Engineering, Vol. 114, $\mathrm{n}^{\circ} 1$, pp. 75-102.

BALOGUN O.S., 1985. — Design of real-time feedback control for canal systems using linear quadratic regulator theory *, $\mathrm{Ph}$. D thesis, Department of Mechanical Engineering, University of California at Davis, 230 p.

BANDARAGODA D.J., BADRUDDIN M., 1992. - * Moving towards demand-based operations in modernized irrigation systems in Pakistan $\curvearrowright$. IIMI Country Paper n ${ }^{\circ} 5,42$ p.

BARBET P., 1990. - « Modèle standard de simulation du fonctionnement des systèmes hydrauliques Barrage-Rivière. Application à la gestion des lachures », CEMAGREF, ENITRTS, $106 \mathrm{p}$.

BAUME J.-P., 1990. - * Régulation des canaux d'irrigation : Etude du sous-système bief avec vanne », CEMAGREF, ENGREF, DEA National d'Hydrologie de I'USTL Montpellier, $170 \mathrm{p}$.

Baume J.-P., Kosuth P., Malaterre P.O., 1992a. - «Régulation des canaux d'irrigation; Notes de cours », CEMAGREF, 60 p.

Baume J.-P., Malaterre P.O., 1992b. - « Design of a decision support tool to improve manual operation procedures of an irrigation canal », CEMAGREF-IIMI International Workshop, Montpellier, October 1992, 10 p.

Baume J.-P., SAlly H., Malaterre P.O., Rey J., 1993. * Development and field-installation of a mathematical simulation model in support of irrigation canal management ". IIMI and CEMAGREF Research paper, 89 p.

BHUTTA M.N., KuNE J.W., 1992, — * Application of a hydraulic model for testing management decisions at distributary level ». Irrigation Science, pp. 15-20.

BORNE P. et al., 1990. - - Commande et optimisation des processus », Editions Technip, 15 ème Paris, 303 p.

BORNE P. et al., 1992. - « Modélisation et identification des processus, tome 2 », Editions Technip, 15ème Paris, 309 p.
BURT C.M., 1983. - Regulation of sloping canals by automatic downstream control », Thèse de Doctorat, Utah State University.

BUYALSKI C.P., SERFozo E.A, 1979. - « Electronic filter level offset (EL-FLO) plus reset equipment for automatic downstream control of canals s, Technical Report REC-ERC-79-3, U.S. Department of Interior, Bureau of Reclamation, Denver Office, $145 \mathrm{p}$.

BUYALSKI C.P., 1991. - «Canal systems automation manual. Volume I , A Water Resources Technical Publication, U.S Department of Interior, Bureau of Reclamation, Denver Office, $113 \mathrm{p}$.

CHAN S.P., YAO B., 1990. - « Performance study of GPC for processes with time delay », IEEE, pp. 384-389.

Chevereau G., 1991. - * Contribution à l'étude de la régulation dans les systèmes hydrauliques à surface libre „, Thèse de Doctorat de I'Institut National Polytechnique de Grenoble, 122 p.

Clemmens A.J., 1987, - * Delivery system schedules and required capacities », dans Zimbelman D.D. (ed.), 1987.

Ceeuret C., 1977. - « Stabilité et précision de la régulation dynamique $»$, La Houille Blanche $\mathrm{n}^{\circ} 2 / 3$, pp. 272-277.

Corriga G., Fanni A., SAnNa S., USAI G., 1982a. - «A constant-volume control method for open channel operation s. International Journal of Modelling \& Simulation, Vol. 2, $\mathrm{n}^{\circ}$ 2, pp. 108-112.

Corriga G., SAnna S., USAI G.. - - Centralized control of irrigation canal systems », date et source inconnues, pp. 6983.

CORRIGA G., SANNA S., USAI G., 1980. - « Frequency response and dynamic behaviour of canal networks with self-levelling gates », Appl. Math. Modelling, Vol. 4, pp. 125-129.

Corriga G., SANNA S., USAI G., 1982b. - « Sub-optimal level control of open-channels $»$, Proceedings International AMSE conference Modelling \& Simulation, Vol. 2, pp. 67-72.

Corriga G., SANNA S., USAI G., 1983. - « Sub-optimal constant-volume control for open channel networks". Appl. Math. Modelling, Vol. 7, pp. 262-267.

DE Leon Mojarro B., 1986. - « Contribution à l'amélioration de la gestion des périmètres irrigués », Thèse de Doctorat, USTL Montpellier, $137 \mathrm{p}$.

DeLtour J.-L., 1988. - « La régulation des systèmes d'irrigation ", DEA, Ecole Nationale Supérieure d'Hydraulique de Grenoble, 87 p.

DELTOUR J.-L., 1992. - «Application de l'automatique numérique à la régulation des canaux; Proposition d'une méthodologie d'étude ». Thèse de Doctorat, Institut National Polytechnique de Grenoble, 153 p.

FALVEY H.T., 1987. - «Philosophy and implementation of Gate Stroking », Proceedings ASCE Portland, pp. 176-179.

FAng Z.X., Voron B., Boquillon C., 1989, - « Programmation dynamique : application à la gestion d'une retenue pour l'irrigation ». Journal Hydrological Sciences 34, 4, pp. 415424.

FILIPOVIC V., MiLoseVIC Z., 1989. - " Dyn ${ }^{2}$ method for optimal control of water flow in open channels ", Journal of irrigation and drainage engineering, Vol. 115, $\mathrm{n}^{\circ} 6$, pp. 973-981.

FLOREA J., POPA R.. - « Optimal operation of an irrigation canal system for unsteady flow conditions $»$, date et source inconnues, pp. 33-45. 
FRAMJ K.K., VeRDIER J., 1978. — «tat de l'art $n^{\circ} I$ : Irrigation. drainage et maîtrise des crues : Chapitre 9: L'automatique dans l'irrigation, pp. 459-578 \%, ICID. New Delhi, 676 p.

FREROT A., 1987, «Procédures d'optimisation des consignes de gestion d'un réseau d'assainissement automatisé ». Thèse de Doctorat, Cergrene, Ecole Nationale des Ponts et Chaussées, 296 p.

GARCIA A., 1988. - * Control and regulation of open channel flow $\%$. Master of Science, University of California, Davis, $232 \mathrm{p}$.

Garcia A., HubBard M., De V ries J.J., 1992. — «Open channel transient flow control by discrete time LQR methods ", Automatica, Vol. $28, \mathrm{n}^{\circ} 2$, pp. 255-264.

GILBERT D., 1990. - "Gestion automatisée en temps réel des réseaux d'eau potable. Calcul des commandes optimales par différentes approches $»$, Thèse de Doctorat, Université de Strasbourg, $132 \mathrm{p}$.

GousSARD J., 1993, - * L'automatisation des réseaux d'irrigation en canaux $»$. Commission Internationale des Irrigations et du Drainage, Groupe de travail sur la Construction, la Réhabilitation et la Modernisation des Projets d'Irrigation, $103 \mathrm{p}$.

Groscl.aude J.-Y., TARdieu H., 1985. - " La gestion automatique des eaux d'étiage - Description et premiers résultats *. pp. 35-39.

Hubbard M., Devries J.J., Balogun O.S., 1987. - « Feedback control of open channel flow with guaranteed stability $»$, AIRH Congrès, pp. 408-413.

HUBERT P., 1984. - a Eaupuscule : Une introduction à la gestion de l'eau », Ellipses, 192 p.

HURAND P., KOSUTH P, 1993. - «Régulations en rivière. La Houille Blanche », $\mathrm{n}^{\circ}$ 2/3 1993, pp. 143-149.

IıмІ, 1989. - - Study on irrigation systems rehabilitation and improved operations and management, Volume 1 », Final report for ADB Regional Technical Assistance, 149 p.

KHALADI A., 1992. — "Gestion automatique des transferts d'eau en réseaux hydrauliques maillés à surface libre: Application au réseau des Wateringues ", Thèse de Doctorat, Ecole Centrale de Lyon, CEMAGREF, 237 p.

Kompass E.J., 1993. — * Dynamic simulation : nothing else will do again for control design », dans Control Engineering, May 1993, Volume 40, $\mathrm{n}^{\circ} 6$, pp. 61-62.

Kosuth P., Rey J., Malaterre P.O., BAUME J.-P., LAMACQ S., 1992. - * Application of mathematical modelling for the improvement of irrigation canal operation ». Proceedings du colloque CEMAGREF-IIMI, Montpellier, France, 26-30 octobre 1992, $335 \mathrm{p}$.

KraAtz D.B., Mahajan I.K., 1982. - « Petits ouvrages hydrauliques $»$, Volumes $26 / 1$ et $26 / 2$, Bulletin FAO d'Irrigation et de Drainage, $307+244 \mathrm{p}$.

KUANYI Z., 1989. - «Contribution à l'automatisation de processus à temps mort $»$. Thèse de Doctorat de l'Université Catholique de Louvain, 168 p.

LaRminat P., 1993. - « Automatique, Commande des systèmes linéaires $\%$, Hermès, 321 p.

LEE T.H., LAI W.C., KWEK K.H., 1990. — * Extended generalised predictive control incorporating feedforward", pp. 27032708.

LEFEBVRE J., 1977. - * La régulation dynamique, sa mise en auvre au canal de Provence $»$, La Houille Blanche, $n^{\circ} 2 / 3$, pp. 265-270.
LESAFFRE B., RIEUL. L., 1992. - * L'irrigation: situation présente et perspectives », C.R. Acad. Agric. Fr., n 6, pp. 35-50.

LINKENS D.A., MAHFOUF M., 1992. - « Generalized predictive control with feedforward (GPCF) for multivariable anaesthesia », Int. J. Control, Vol. 56, n 5, pp. 1039-1057.

LIU F., FEYEN J., BERLAMONT J., 1992. — * Computation method for regulating unsteady flow in open channels . Journal of irrigation and drainage engineering, Vol. $118, \mathrm{n}^{\circ} 10, \mathrm{pp} .674-$ 689.

MALATERRE P.O., 1989. - « Etude des possibilités d'amélioration des règles de gestion manuelle du canal principal rive droite de Kirindi Oya grâce à un modèle mathématique de simulation des écoulements », CEMAGREF, ENGREF, IIMI. DEA National d'Hydrologie USTL Montpellier, 121 p.

MALATERRE P.O., 1991. - « Water flow regulation in irrigation canals: present methods and perspectives $»$. Séminaire IIMI, CEMAGREF Montpellier Division Irrigation, 7 p.

MALATERRE P.O., 1994. - Modélisation, analyse et commande optimale $L Q R$ d'un canal d'irrigation ». Thèse de Doctorat, ENGREF - CEMAGREF - LAAS CNRS, 200 p.

MARZOUKI T.Z., 1989. — « Le système hydraulique de la rivière larcis (barrages, rivière, perimètres irrigués). Modélisation hydraulique, analyse et amélioration de la gestion „, CEMAGREF, ENGREF, CARA, 63 p.

MERRIAM J.L., 1992. - « The need on the farm for a flexible water supply schedule », Colloque « Advances in Planning, Design and Management of Irrigation Systems as Related to Sustainable Land Use », Louvain, 14-17 septembre 1992 , édité par Feyen et al., pp. 147-156.

NАлм K., 1981. - * Application des automates à structure variable à la commande d'un canal d'irrigation ». Automatique Systems analyis and control, Vol. 15, n 3, pp. 263-270.

O'Loughlin E.M., 1972. - * Application of unsteady flow analysis to operation decisions in long aqueducts $\$$, Rapport 16, Question 28-2, VIIIème Congrès de LA CIID, Bulgarie.

PARENT E., 1991. - - Elaboration des consignes de gestion des barrages réservoirs ", Thèse de Doctorat ENPC-Cergrene.

PERRIN G., 1989. - * Automatisation d'un système d'irrigation à gestion centralisée », Conservatoire National des Arts et Métiers, Centre Régional Associé de Metz, 154 p.

Piquereau A., Tardieu H., Verdier J., Villocel A., 1984. * Réduction des pertes en eau par l'automatisation de la gestion d'un barrage realimentant une rivière en vue de l'irrigation », Communication au XIIème congrès de la CIID, $16 \mathrm{p}$.

PIQUereau A., VIllocel A., 1982. — Gestion automatique des eaux d'étiage; Cas de la rivière Arrats», ONERA, CERT/DERA Toulouse, CACG, 125 p.

PluSQUElLEC H., 1988. - * Improving the Operation of Canal Irrigation Systems. An Audiovisual Production », The Economic Development Institute and the Agriculture and Rural Development Department of the World Bank, 154 p.

Plusquellec H., Burt C., Wolter H.W., 1994. - * Modern Water Control in Irrigation », World Bank Technical Paper Number 246, Irrigation and Drainage Series, 104 p.

Ramis E., Deschamps C., Odoux J., 1979. - « Cours de Mathématiques Spéciales - Tome 1: Algèbre », Masson, 436 p.

REDDY J.M., 1986. - « Evaluation of optimal constant volume control for irrigation canals ». ASAE, 14 p.

REDDY J.M., 1990. - « Local optimal control of irrigation canals $»$, Journal of Irrigation and Drainage Engineering, Vol. 116, $\mathrm{n}^{\circ} 5$, pp. 616-631. 
REDDY J.M., 1992a. - "Decentralized constant-volume control of irrigation canals », Colloque « Advances in Planning, Design and Management of Irrigation Systems as Related to Sustainable Land Use ", Louvain, 14-17 septembre 1992, édité par Feyen et al., pp. 889-898.

REDDY J.M., DiA A., Oussou A., 1992b. — « Design of control algorithm for operation of irrigation canals *, Journal of Irrigation and Drainage Engineering, Vol. 118, $\mathrm{n}^{\circ} 6$, pp. $852-$ 867.

REY J., 1990. - « Contribution à la modélisation et la régulation des transferts d'eau sur des systèmes de type rivière/baches intermédiaires ». CEMAGREF, ENGREF, DEA National d'Hydrologie USTL Montpellier, $80 \mathrm{p}$.

Rey J., Malaterre P.O., Baume J.P., 1993. - " Use of a simulation model to improve the manual operation procedures of an irrigation canal (Kirindi Oya - Sri Lanka) „, Proceedings Asian Regional Symposium Beijing, HR Wallingford, session 3, pp. 27-40.

Rodellar J., Gomez M., Bonet L., 1993. - « Control method for on-demand operation of open-channel flow s, Journal of Irrigation and Drainage Engineering, Vol. 119, $\mathrm{n}^{\circ} 2$, pp. $225-$ 241.

Roux A., 1992. - *Gestion des étiages de rivière par les méthodes de l'automatique. Application à la rivière Dropt », CEMAGREF, ENITRTS, 177 p.

SABet M.H., CoE J.Q., RAmirez H.M., Ford D.T., 1985. *Optimal operation of California Aqueduct », Journal of Water Ressources Planning and Management, Vol. 111, $n^{\circ} 2$, pp. 222-237,

SANFILIPPO F., 1993. - «Application du régulateur PIR au cas des canaux à forte pente », DEA de l'Université Claude Bernard, Lyon 1, 137 p.

SAWAdOGo S., 1992a. - Modélisation, commande prédictive et supervision d'un système d'irrigation ». Thèse de Doctorat, LAAS-CNRS Toulouse, $152 \mathrm{p}$.

SaWAdogo S., Achaibou A.K., Aguilar-Martin J., 1991a. *An application of adaptative predictive control to water distribution systems », IFAC, ITAC 91, Singapour, 6 p.

Sawadogo S., Achaibou A.K., Aguilar-Martin J., 1992b. "Long-range predictive control of an hydraulic systems", CEMAGREF-IIMI Workshop, 9 p.

Sawadogo S., Achaibou A.K., Aguilar-Martin J., MoraCAMINO F., 1991b. - - Output tracking by inverse adaptive control : application to water distribution systems $»$ IMACS IFAC, Lille, Proceedings, Vol 1, pp. 88-93.

Sawadogo S., Achaibou A.K., Aguilar-Martin J., MoraCAMINO F., 1992c. - * Intelligent control of large water distribution systems : a two level approach», SICICI 92, Singapour, Proceedings, pp. 1085-1089.

Sawadogo S., Malaterre P.O., Kosuth P., 1994. - « Multivariable optimal control for on-demand operation of irrigation canals », International Journal of Systems Science, 30 p., accepted to be published.
SCHULTZE F.E., 1989. - - Concepts in irrigation management and the related research issues $»$. IIMI Pakistan, $14 \mathrm{p}$.

SChuUrmans J., 1991. - * Models and controllers of open channel flow for irrigation purposes: A literature study", Delft University of Technology, $46 \mathrm{p}$.

SChuURmans J., 1992. — * Controller Design for a Regional Downstream Controlled Canal », Delft University of Technology, Laboratory for Measurement and Control, 100 p.

SHAND M.J., 1971. - «Automatic downstream control systems for irrigation canals ", Thèse de Doctorat, Université de Californie, Berkeley, 159 p.

Shinskey F.G., 1988. - « Process control systems, Application, Design, and Tuning ». McGraw-Hill Publishing Company, New York, 526 p.

SOETERBOEK R., 1990. - «Predictive control: a unified approach ». Thèse, Delft University of Technology, 358 p.

TARDIEU H., 1988. - «Automatic dam management and river regulation for irrigation purposes ». Irrigation and Drainage Systems, $n^{\circ} 2$, pp. 53-61.

Tомісіс B., 1989. - « A general optimization module for realtime control of surface water resources », Master of Science, The International Institute for Hydraulic and Environmental Engineering, Delft University, 174 p.

Trouvat J.-L., 1991, "Contribution à une meilleure gestion des rivières de Gascogne», CEMAGREF, CACG, ENGREF, $61 \mathrm{p}$.

USBR, 1979. - « Gate Stroking ». Internal paper, 15 p.

VALERA A., 1991. - " Design-Management interactions of Malaysia's Kerian irrigation scheme », IIMI Country Paper $n^{\circ} 1$, $64 \mathrm{p}$.

VALIRON F., 1984. - « Gestion des Eaux. Principes, moyens, structures », Presses de l'Ecole Nationale des Ponts et Chaussées, 343 p. (POM)

VALIRON F., 1988. - «Gestion des Eaux. Automatisation, informatisation, télégestion », Presses de l'Ecole Nationale des Ponts et Chaussées, 551 p.

VERDIER J., 1986. - "Informatisation de la commande du transport et de la distribution d'eau d'irrigation », XIVè Journées régionales européennes de la CIID, La Manga del Mar Menor (Espagne), 15 p.

VERDIER J., MiLlo J.-L., 1992. — * Maintenance des périmètres irrigués », Collection Techniques Rurales en Afrique, Ministère de la Coopération et du Développement, 323 p.

ZIHUI L., MANZ D.H., 1992. - «Optimal operation of irrigation canal systems using nonlinear programming - dynamic simulation model ». CEMAGREF-IIMI International Workshop, Montpellier, October 1992, pp. 297-306.

ZIMBELMAN D.D., 1987. — * Planning, operation, rehabilitation and automation of irrigation water delivery systems *, Proceedings of a symposium ASCE, Portland, Oregon, USA, 28-30 juillet $1987,377 \mathrm{p}$. 


\section{BON DE COMMANDE :}

NOM :

ORGANISME :

ADRESSE :

\section{JOURNEES DE L'HYDRAULIQUE :}

11 èmes. Utilisation des ressources en eau d'un bassin dans le cadre de l'aménagement du territoire (1970) 12èmes. Hydrotechnique des liquides industriels (1972)...

14èmes. La mécanique des Fluides et l'environnement (1976).

15èmes. L'Hydrotechnique au service d'une politique de l'Eau (1978)

16èmes. Rejets de chaleur à l'atmosphère (1980)

17 èmes. L'assainissement de demain (1982)

18 èmes. L'Hydraulique et la maîtrise du Littoral (1984)

19 èmès. L'Impact des activité humaines sur les eaux continentales (1986)

20èmes. Machines hydrauliques (1989)

21èmes. Les Eaux souterraines et la gestion des eaux (1991)

22èmes. L'Avenir de l'Eau (1992)

23èmes. Crues et Inondations (1994)

$800 \mathrm{~F}$

$800 \mathrm{~F}$

$800 \mathrm{~F}$

$800 \mathrm{~F}$

$800 \mathrm{~F}$

$800 \mathrm{~F}$

$800 \mathrm{~F}$

épuisé

$800 \mathrm{~F}$

$1000 \mathrm{~F}$

$1000 \mathrm{~F}$

$1000 \mathrm{~F}$

\section{COLLOQUES D'HYDROTECHNIQUE :}

Législation des petites centrales - France (1991)
Les Sécheresses (1989)

Les modèles mathématiques pour la gestion de la qualité des eaux superficielles (1989)

Economie de l'hydrologie urbaine (1990)

Formation de l'Ingénieur hydraulicien européen (1991)

Stockage de l'eau et maîtrise des débits (1991)

Cavitation (1992)

Irrigation 2000 (1992)

Crues et Laves torrentielles (1992)

La pluie, source de vie, choc de pollution (1993).

Bruit et Vibrations des Pompes (1993).

Transports solides en eaux continentale et littorale (1993)

Mesure et contrôle de débits en conduite (1994)

Eaux minérales et thermales (1994)

Glaciologie et nivologie - Etat des recherches et des connaissances à la fin du XXè s.(1995)

\section{GUIDES :}

Prévision des crues (1987)

Conception, réalisation et exploitation des petits aménagements hydroélectriques (1985).

.édition française

.édition anglaise

\section{CAHIERS DES CHARGES - TYPES :}

Pour la fourniture de turbines hydrauliques :

1-turbines Francis, Pelton et Kaplan, +

spécifications propres aux turbines Francis (1972)

2-spécifications propres aux turbines Pelton (1972)

3-spécifications propres aux turbines Kaplan (1973)

4-groupes du type Bulbe (1974)

.édition française

.édition anglaise

Pour la fourniture et le montage des conduites forcées en acier et de leurs accessoires (1972)

Données techniques annexes (1968)

épuisé

épuisé

$600 \mathrm{~F}$

épuisé

épuisé

$1000 \mathrm{~F}$

$1000 \mathrm{~F}$

$500 \mathrm{~F}$

$800 \mathrm{~F}$

$800 \mathrm{~F}$

$600 \mathrm{~F}$

$600 \mathrm{~F}$

$600 \mathrm{~F}$

$500 \mathrm{~F}$

$600 \mathrm{~F}$

$600 \mathrm{~F}$

$100 \mathrm{~F}$

$600 \mathrm{~F}$

$200 \mathrm{~F}$

$200 \mathrm{~F}$

$600 \mathrm{~F}$

$800 \mathrm{~F}$

$400 \mathrm{~F}$

T.V.A. 5,5\% incluse pour la France

Frais de port en sus 


\section{GLACIOLOGIE ET \\ NIVOLOGIE \\ (1 ${ }^{\text {ère }}$ partie)}

d'après le colloque "Glaciologie et nivologie :

état des recherches et connaissances à la fin $d u X X^{e}$ siècle" organisé par la Société hydrotechnique de France à Grenoble, les 15 et 16 février 1995 


\section{Allocution d'ouverture du colloque « Glaciologie et nivologie »}

Chers amis hydrauliciens

La SOCIÉTÉ HYDROTECHNIQUE DE FRANCE existe depuis 80 ans et, depuis plus de 60 ans a établi son rythme de fonctionnement actuel de trois manifestations publiques annuelles, incluant, toutes les années paires, un congrès plus solennel portant le nom de «Journées de l'Hydraulique ".

L'année 1994 qui avait vu se dérouler dans cette ville de Grenoble un colloque prospectif sur la mesure des débits en conduites s'est terminée par un colloque à Paris sur les Eaux Minérales et Thermales, qui a permis à tous les spécialistes des eaux souterraines, très actifs dans notre société, de rencontrer les scientifiques d'une branche particulièrement vivante du monde médical et les responsables techniques et commerciaux de la considérable industrie des eaux en bouteille. Ce colloque suivait de peu notre congrès biennal, constituant les $23^{\text {emes }}$ Journées de 1'Hydraulique sur le thème des "Crues et Inondations", organisé à Nîmes en septembre, avec le succès qu'on peut imaginer compte tenu du caractère très actuel, très médiatique, du sujet et du caractère symbolique de la ville qui nous accueillait.

Aujourd'hui, nous sommes à Grenoble pour ce colloque de glaciologie et de nivologie. En septembre, nous organisons avec le Ministère de l'Environnement, en complément de notre traditionnel calendrier triannuel, une journée d'information sur les SDAGE, ces «Schémas Directeurs pour l'Aménagement et la Gestion des Eaux » qui jouent un rôle essentiel dans la nouvelle politique de l'eau, puis nous reprendrons notre rythme avec, en octobre, un colloque d'hydrologie urbaine en copatronnage avec l'AGHTM et en novembre un colloque sur la "Connaissance et la valorisation des nappes profondes ». Nous avons en préparation pour 96 , un colloque sur le prix de l'eau, en coopération avec l'AFPE, un colloque sur les machines hydrauliques et les $24^{\text {èmes }}$ journées de l'hydraulique sur « L'Eau, 1'Homme et la Nature » montrant le rôle que peuvent avoir les sciences hydrotechniques dans une perspective de développement durable.

Cette présentation rapide du programme de ces trois années montre à la fois la continuité de l'activité S.H.F. et la diversité des domaines abordés.

Ces objectifs ne peuvent être atteints que grâce au travail régulier de plus de 20 groupes spécialisés, qui, regroupés au sein de 3 divisions, constituent l'armature active du Comité Scientifique et Technique dont j'ai l'honneur d'assurer la présidence.
Vous trouverez sur les tables de l'accueil, l'organigramme complet de ce Comité et de ses trois divisions :

- l'une, fondamentale, sous le nom de «Mécanique des Fluides »

- l'autre, sous le nom d' « Applications industrielles de la Mécanique des fluides ", se rattache aux origines lointaines de la Société, née avec la Houille Blanche, qui a localisé notre revue, la troisième appelée désormais l'Eau, soustitrée « ressources, aménagement, environnement " souligne l'expansion du domaine hydrologique et des sciences de l'eau dans la nature.

C'est à cette division que se rattache la très importante Section "Glaciologie, Nivologie ", qui a entièrement construit le colloque auquel vous allez participer pendant ces deux journées grenobloises. Je tiens à rendre hommage à la très haute qualité du travail de cette section, longtemps animée par Monsieur de CRECY qui nous présentera tout à l'heure l'ANEMA dont il fut l'un des principaux créateurs et actuellement coanimée par Messieurs DUBAND, FEUVRIER et REYNAUd qui illustrent l'union dans l'action de la Division technique Générale d'E.D.F., du CEMAGREF et de l'Université.

Cette section constitue à elle seule une véritable société savante, avec sa vie propre, son action fédérative, sa liaison avec les organismes internationaux, son organisation particulière de journées glaciologiques, qui contribuent à maintenir Grenoble au rang de capitale, au moins française, de la Glaciologie.

Grâce à son rayonnement propre, et à sa liaison active avec I.G.S., la Section «Glaciologie » a montré que pour ces deux jours un véritable congrès international auxquels participent souvent comme auteurs de communications, des spécialistes de haut rang que nous sommes heureux d'accueillir venus de Suisse, d'Espagne, d'Italie et d'Allemagne.

Merci à tous ceux qui ont organisé ce colloque et à tous ceux qui vont l'animer, et lui apporter leur précieux concours.

Monsieur DUBAND a assuré la liaison avec le Bureau S.H.F. pour l'organisation de ce colloque.

P. Cazenave

Président du Comité scientifique et technique de la S.H.F. 